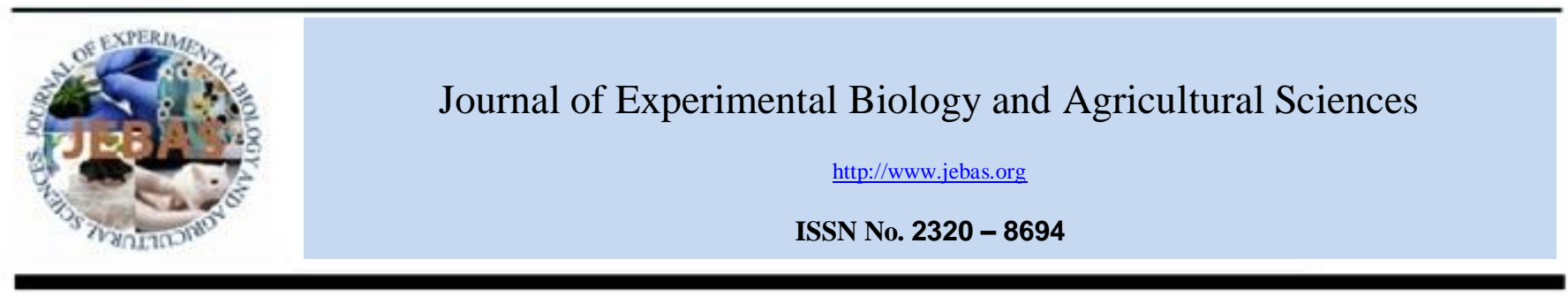

\title{
IMPACT OF GEOGRAPHIC'S VARIATION ON THE ESSENTIAL OIL YIELD AND CHEMICAL COMPOSITION OF THREE Eucalyptus SPECIES ACCLIMATED IN TUNISIA
}

\section{Elaissi Ameur ${ }^{1 *}$, Medini Hanene ${ }^{1}$, Rouis Zied ${ }^{2}$, Khouja Mohamed Larbi ${ }^{3}$, Chemli Rachid ${ }^{1}$ and Harzallah-Skhiri Fethia ${ }^{1}$}

${ }^{1}$ Laboratory of The Chemical, Galenic and pharmacological Drug Development, Faculty of Pharmacy, University of Monastir, Avenue Avicenne, 5019 Monastir, Tunisia

${ }^{2}$ Laboratory of Genetic, Biodiversity and Bio-resources Valorisation, Higher Institute of Biotechnology of Monastir, University of Monastir, Avenue Tahar Haddad, 5000 Monastir, Tunisia

${ }^{3}$ National Institute for Research on Rural Engineering, Water and Forestry, Institution of Agricultural Research and Higher Education, BP. N.2, 2080 Ariana, Tunisia

Received - March 28, 2015; Revision - April 10, 2015; Accepted - June 15, 2015

Available Online - July 07, 2015

DOI: http://dx.doi.org/10.18006/2015.3(3).324.336

\begin{abstract}
KEYWORDS
Eucalyptus

Essential oils

1,8 -cineole

$\mathrm{ACP}$

HCA

GC-MS

ABSTRACT

Present study has been carried out to estimate the impact of geographical distribution on the yield and chemical constitute of three Eucalyptus verities viz E. cinerea F. Muell. ex Benth., E. astringens Maiden and E. sideroxylon A.Cunn. ex Schauer-. These species were collected from six arboreta of Tunisia in January 2008. The essential oil was extracted by hydrodistillation method and estimated the essential oil yield which varies from $1.5 \pm 0.1 \%$ to $4.0 \pm 0.2 \%$. Results of the study revealed that yield of essential oil are not only depends on the Eucalyptus species but also depends on the origin of harvest. $E$. sideroxylon A. Cunn. exWoolls, cultivated in jbel abderrahman arboreta and E. cinerea F. Muell. ex Benth. from choucha (sejnanae) arboreta provided the lowest and the highest percentage of essential oil amongst all the studied provenances, respectively. GC (RI) and GC/MS analysis showed the presence of 163 components, representing 98.8 to $99.5 \%$ of the total oil. The contents of the different samples varied according to the species and the origin of harvest. The main components of the Eucalyptus essential oil were 1,8-cineole $(39.1 \pm 0.0-79.4 \pm 0.0 \%)$, followed by $\alpha$-pinene $(2.1 \pm 0.0-30.0 \pm 0.0)$, trans-pinocaveol
\end{abstract}

* Corresponding author

E-mail: aelaissi@yahoo.fr (Elaissi Ameur)

Peer review under responsibility of Journal of Experimental Biology and Agricultural Sciences.

Production and Hosting by Horizon Publisher (www.myvision.webs.com/horizon.html).

All rights reserved.
All the article published by Journal of Experimental

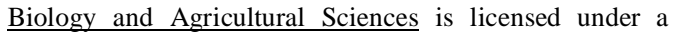
Creative Commons Attribution-NonCommercial 4.0 International License Based on a work at www.jebas.org.

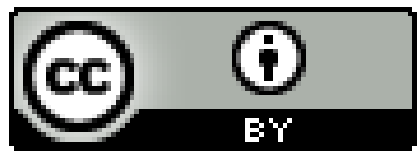




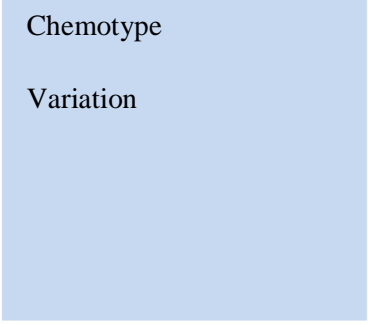

$(0.7 \pm 0.5-10.0 \pm 2.5 \%), \alpha$-terpineol $(0.2 \pm 0.0-8.8 \pm 1.1 \%)$, globulol $(0.3 \pm 0.0-5.7 \pm 1.3)$, aromadendrene $(0.2 \pm 0.0-4.9 \pm 2.1 \%)$, limonene $(0.6 \pm 0.0$ - $4.3 \pm 0.8 \%)$, pinocarvone $(0.2 \pm 0.0-3.7 \pm 0.6 \%)$, bicyclogermacrene $(0.0-3.1 \pm 0.0 \%), \alpha$-terpinyl acetate $(0.0-2.9 \pm 0.8 \%), \quad p$-cymene $(0.7 \pm 0.0$ $2.7 \pm 2.1 \%), \beta$-carophyllene $(0.3 \pm 0.0-1.9 \pm 0.0 \%)$, viridiflorol $(0.2 \pm 0.0-1.8 \pm 0 . \%)$, epiglobulol (traces $1.3 \pm 0.6 \%)$ and spathulenol $(0.2 \pm 0.0-1.1 \pm 0.0 \%)$. The principal component and the hierarchical cluster analyses separated the Eucalyptus populations leaf essential oils into four groups, each constituting a chemotype. E. sideroxylon from Korbous and Sejnane arboreta afforded the highest mean percentage in 1,8-cineole.

\section{Introduction}

Genus Eucalyptus comprises about 900 species and subspecies (Pereira et al., 2014). More than 300 species of this genus contain volatile oil in their leaves. In 1957, total 117 Eucalyptus have been introduced in Tunisia. They were used essentially as fire wood, for the production of mine wood and against the erosion (Khouja et al., 2001). However, less than 20 species have ever been exploited commercially for the production of essential oil rich in 1,8-cineole (>70\%) which is essentially used in the pharmaceutical and cosmetic industries (Pino et al., 2002). In Tunisian folk medicine, inhalation of Eucalyptus sp. essential oil has been traditionally used to treat respiratory tract disorders such as pharyngitis, bronchitis and sinusitis (Boukef, 1986). Many studied have been demonstrated their antibacterial, antifungal and antiviral activities of Eucalyptus sp. essential oil against a wide range of microorganisms (Su et al., 2006;Cermelli et al., 2008; Gilles et al., 2010; Jha et al., 2014). Furthermore, allelopathic effect of this essential oil against many weeds was also reported by many researchers (Batish et al., 2004; Verdeguer et al., 2009; Rassaeifar et al., 2013). Eucalyptus essential oil was also reported as an effective anti-inflammatory, analgesic, antioxidant, antimutagenic, insecticide, nematocide and acaricide oil (Batish et al., 2008; Bugarin et al., 2014; Rossi \& Palacios, 2015).The Eucalyptus essential oils can be obtained by different methods, such as hydrodistillation, supercritical $\mathrm{CO}_{2}$ extraction, microwave-assisted extraction, and vacuum extraction by solvents. These methods affect the final yield of the oil and it varied from $0.1 \pm 0.1$ to $5.7 \pm 0.57 \%$ (Elaissi et al., 2010a; Elaissi et al., 2010b). High levels of 1,8-cineole $(87.8 \%)$ were found for the majority of the Eucalyptus species (Zrira et al., 2004; Elaissi et al., 2010c). Generally, the yields and composition of the oils varies and it depending on species, used part, plant origin zone, collection period, growth stage of the plant as well extraction methods and storage conditions (Arango-Bedoya et al., 2012).

In previous studies published on Eucalyptus species growing in Tunisia, characterization of the leaves essential oils of 48 Eucalyptus species and their antibacterial activities were carried out by the same authors. On the basis of this work three Eucalyptus species, E. cinerea F. Muell. ex Benth., E. astringens Maiden and E. sideroxylon A.Cunn. ex Schauer, which demonstrated the best antibacterial activity and also the best content in essential oil yield and 1,8-cineole, were select for this research work to identify the effect of the origin of harvest on their essential oils yield and composition.

\section{Materials and Methods}

\subsection{Plant Materials}

Clean and mature leaves of three Eucalyptus species L Hér., viz, E. astringens Maiden, E. cinerea F. Muell. ex Benth. and E. sideroxylon A.Cunn. ex Schauer, were collected from six arboreta belonging to lower humid, higher humid and subhumid bioclimatic stage of Tunisia in January 2008, Table 1. The leaves were dried in airy premises, shielded from the light, then packed in paper bags, and kept in the shade. Botanical voucher specimens of the collected species have been deposited in the Herbarium of the Pharmacognosy Laborotary, Faculty of Pharmacy, Monastir, Tunisia (references 0156, 0157, 0158, 0159, 0160, 0.161, 0.162, 0.163, 0.164, 0.165, 0166 and 0167$)$.

\subsection{Sample preparation and extraction of Essential Oils}

The essential oils were extracted by hydrodistillation method, for this $100 \mathrm{~g}$ of boorishly crushed Eucalyptus leaves for $4 \mathrm{~h}$, using a standard apparatus recommended in the European Pharmacopoeia. Hydrodistillations were performed in triplicate for each Eucalyptus species. The oil collected from each plant was dried $\left(\mathrm{Na}_{2} \mathrm{SO}_{4}\right)$ and stored at $4{ }^{\circ} \mathrm{C}$ until analysis. The yield of essential oil was expressed in \% $(\mathrm{v} / \mathrm{w})$ of the dry material (Elaissi et al., 2010a; Elaissi et al., 2010b).

\subsection{Chemical analysis}

\subsubsection{GC Analysis}

Quantative and qualitative data of the essential oils were determined in triplicate by GC and GC/MS, respectively. GC Analysis was carried out with a Hewlett-Packard 6890 apparatus equipped with FID and a polar Carbowax column (30 m_0.32 mm i.d., film thickness $0.25 \mathrm{~mm}$ ). The oven temperature was programmed isothermal at $35^{\circ} \mathrm{C}$ for $1 \mathrm{~min}$, rising from 35 to $250^{\circ} \mathrm{C}$ at $5^{\circ} \mathrm{C} / \mathrm{min}$, and then held isothermal at $250^{\circ} \mathrm{C}$ for $3 \mathrm{~min}$; injector temp., $250^{\circ} \mathrm{C}$; detector temp., $280^{\circ} \mathrm{C}$; $\mathrm{N} 2$ used as carrier gas $(1.2 \mathrm{ml} / \mathrm{min})$. 
Table 1 Provenances and Classification by Means of Duncan_s Multiple Range Test of the Average Essential Oil Yields of 3 Eucalyptus Species

\begin{tabular}{|c|c|c|c|c|c|c|c|c|c|}
\hline $\begin{array}{l}\text { Eucalyptus } \\
\text { species }\end{array}$ & $\begin{array}{c}\text { Provenanace } \\
\text { (Arboreta) }\end{array}$ & Latitude & Longitude & $\begin{array}{l}\text { Altitude } \\
\text { (m) }\end{array}$ & $\begin{array}{l}\text { Annual } \\
\text { rainfall } \\
(\mathrm{mm})\end{array}$ & $\begin{array}{c}\text { Bioclimatique } \\
\text { stage }\end{array}$ & Soil type & Abbreviation & Yield [\%] \\
\hline E. cinerea & Souiniat & $35^{\circ} 54^{\prime}$ & $8^{\circ} 48^{\prime}$ & 492 & 1140 & $\begin{array}{l}\text { humid } \\
\text { inferior with } \\
\text { temperate } \\
\text { winter }\end{array}$ & $\begin{array}{c}\text { sandstone } \\
\text { hydromorphe }\end{array}$ & cin Soui & $\begin{array}{l}3.7 \pm 0.7 \\
\left({ }^{\mathrm{de}}\right)^{\mathrm{a})}\end{array}$ \\
\hline E. cinerea & Zerniza & $30^{\circ} 94$ & $9^{\circ} 7^{\prime}$ & 60 & 920 & $\begin{array}{c}\text { humid } \\
\text { inferior with } \\
\text { warm winter }\end{array}$ & $\begin{array}{c}\text { sandstone } \\
\text { hydromorphe }\end{array}$ & $\operatorname{cin}$ Zer & $\begin{array}{c}3.8 \pm 0.4 \\
\left({ }^{\mathrm{de}}\right)\end{array}$ \\
\hline E. cinerea & Sejnane & $37^{\circ} 3^{\prime}$ & $9^{\circ} 14^{\prime}$ & 159 & 871 & $\begin{array}{c}\text { humid } \\
\text { inferior with } \\
\text { temperate } \\
\text { winter }\end{array}$ & sandstone & cin Sej & $4.0 \pm 0.2\left({ }^{\mathrm{e}}\right)$ \\
\hline E. astringens & Mrifek & $37^{\circ} 07^{\prime}$ & $9^{\circ} 15^{\prime}$ & 60 & 950 & $\begin{array}{l}\text { humid } \\
\text { inferior with } \\
\text { mild winter }\end{array}$ & Marl & ast Mri & $3.1 \pm 1.0\left(^{\mathrm{c}}\right)$ \\
\hline E. astringens & korbous & $36^{\circ} 50^{\prime}$ & $10^{\circ} 35^{\prime}$ & 180 & 540 & $\begin{array}{c}\text { sub-humid } \\
\text { with warm } \\
\text { winter } \\
\end{array}$ & Sandy & ast kor & $3.2 \pm 0.1\left(^{c}\right)$ \\
\hline E. astringens & $\begin{array}{l}\text { Pryor } \\
\text { Choucha } \\
\text { (Sejnane) }\end{array}$ & $37^{\circ} 3^{\prime}$ & $9^{\circ} 14^{\prime}$ & 159 & 871 & $\begin{array}{l}\text { humid } \\
\text { inferior with } \\
\text { temperate } \\
\text { winter }\end{array}$ & sandstone & ast Sej & $\begin{array}{c}3.3 \pm 0.3 \\
\left({ }^{\mathrm{cd}}\right)\end{array}$ \\
\hline $\begin{array}{l}\text { E. } \\
\text { sideroxylon }\end{array}$ & Mrifek & $37^{\circ} 07^{\prime}$ & $9^{\circ} 15^{\prime}$ & 60 & 950 & $\begin{array}{l}\text { humid } \\
\text { inferior with } \\
\text { mild winter }\end{array}$ & Marl & sid Mri & $2.6 \pm 0.3\left(^{b}\right)$ \\
\hline $\begin{array}{l}\text { E. } \\
\text { sideroxylon }\end{array}$ & $\begin{array}{l}\text { Jbel } \\
\text { Abderrahmane }\end{array}$ & $36^{\circ} 40^{\prime}$ & $10^{\circ} 40^{\prime}$ & 255 & 600 & $\begin{array}{c}\text { Sub-humid } \\
\text { with warm } \\
\text { winter }\end{array}$ & sandy clay & $\operatorname{sid} J B A$ & $1.5 \pm 0.1\left({ }^{\mathrm{a}}\right)$ \\
\hline $\begin{array}{l}\text { E. } \\
\text { sideroxylon }\end{array}$ & Korbous & $36^{\circ} 50^{\prime}$ & $10^{\circ} 35^{\prime}$ & 180 & 540 & $\begin{array}{c}\text { sub-humid } \\
\text { with warm } \\
\text { winter } \\
\end{array}$ & Sandy & sid Kor & $2.3 \pm 0.1\left(^{b}\right)$ \\
\hline $\begin{array}{l}\text { E. } \\
\text { sideroxylon }\end{array}$ & Souiniat & $35^{\circ} 54^{\prime}$ & $8^{\circ} 48^{\prime}$ & 492 & 1140 & $\begin{array}{c}\text { humid } \\
\text { inferior with } \\
\text { temperate } \\
\text { winter }\end{array}$ & $\begin{array}{c}\text { sandstone } \\
\text { hydromorphe }\end{array}$ & Sid Soui & $2.6 \pm 0.4\left(^{b}\right)$ \\
\hline
\end{tabular}

a) Yields with different letters in parentheses differ significantly by Duncan_s multiple range test $(\mathrm{p}<0.05)$.

The injected volume was $1 \mathrm{ml}(10 \%$ essential oil in purified hexane). The relative concentration was calculated using the software HP Chemstation, which allows assimilating the percentages of the peak areas to the percentages of the various constituents. Retention indices were obtained by running a series of aliphatic hydrocarbons (C9 - C28) by increasing the number of carbon atoms in the Carbowax column (Elaissi et al., 2012) GC column).

\subsubsection{GC/MS Analysis}

The essential oils were analyzed with a Hewlett-Packard 5890 series II apparatus equipped with a 5972 mass-selective detector and polar Carbowax Column (30 m_0.32 mm i.d., 2.3.4 Statistical Analysis film thickness $0.25 \mathrm{~mm}$ ) in which Helium gas was used as a carrier. The mass spectrometer operating conditions were: 70 $\mathrm{eV}$ ionization voltage, $70 \mathrm{eV} ; 230^{\circ} \mathrm{C}$ ion source. The GC analysis conditions were as described above (see GC Analysis) (Elaissi et al., 2012).

\subsubsection{Compound Identification}

Isolated compounds were based on the comparison of their RI (determined rel. to the tR of n-alkanes (C9-C28)) and mass spectra with those of authentic compounds by means of NBS75K.L. and Wiley 275 databases and with the literature data (Wiley \& Sons, 1998) . 
The data were analyzed using analysis of variance (ANOVA), and the significance of the differences between means was determined at $\mathrm{p}<0.05$ using Duncan's multiple range test. To evaluate the identified essential oil constituents useful for chemical reaction between selected species, 16 compounds were identified from the oil samples with contents in the essential oils of $0.9 \%$ in at last one species, were subjected to PCA and HCA using SPSS 12.0 software (SPSS Inc. Chicago, IL, USA) (Elaissi et al., 2012).

\section{Results and Discussion}

\subsection{Oil Yields}

The Analysis of variance (ANOVA) indicated that the oil yields were significantly different between species to species and arboreta to arboreta $(\mathrm{p}<0.05)$. The analysis of results showed the presence of five overlapping groups (Table 1) ranging from $1.5 \pm 0.1 \%$ for E. sideroxylon cultivated in Jbel Abderrahmane arboreta to $4.0 \pm 0.2 \%$ for E. cinerea grown in Pryor Choucha (Sejnane) arboreta (Table 1), which differed from the others arboreta (Souiniat and Zerniza) by a sandstone soil and by the lowest annual plyviometry. Results of the study also revealed that E. sideroxylon harvested from Souiniat and Mrifek arboreta, which were characterised by a higher annual raining, higher altitude and by a sandstone soil, produced a higher mean percentage of essential oils relatively to those collected from Jbel Abderrahman and Korbous arboreta, while E. astringens was not affected by the environnemental conditions. These result was confirmed by Dunlop et al. (2000) who found that the essential oil yields of some clones of $E$. camaldulensis was not dependent on the sites at which they were grown but rather on their genetic constitution, however Bhatti et al. (2007) reported that E. cerebra essential oil yield varied significantly among different district of PunjabPakistan. The increasing level of essential yield was depend to environnemental conditions such as dry, warm, altitude (Tsiri et al. 2003; Arango-Bedoya et al., 2012; Hassiotis et al., 2014). Furthermore Zrira et al. (1994) reported that E. sideroxylon from morocco, region of Jbilet was richer in essential oil $(2.06 \%)$ than that of the region of Tekerkoust $(1.36 \%)$, while E. astringens from Tekerkoust was the richest one $(2.45 \%)$ against $2.20 \%$ for the other provenances. These results were also proved by Grbovic et al. (2010) which were found that the leaves essential oil yield of E. camaldulensis Dehn. collected from five regions of the coastline of Montenegro varied from $0.67 \%$ for those collected from Bar region to $1.59 \%$ for the region of Tivat. Similarly LI \& Madden (1995) found that the essential oils obtained from leaves of $E$. regnans, E. deligatensis, E. nitens, E. denticulata and $E$. gobulus grown in eight arboreta varied significantly within species and within provenances. It was also demonstrated that E. cinerea from Morocco (Zrira et al., 2004) was poor in essential oils $(0.26 \%)$ than those cultivated in India (2.55 to $2.87 \%$ ) (Kiran Babu \& Singh, 2009).

\subsection{Chemical composition}

The chromatographic analyses (GC (RI) and GC/MS) of the essential oils allowed the identification of 163 compounds (Table 2), representing $98.8-99.5 \%$ of the total oil. Their mean percentage varied within species and harvest zone. The identified components were divided in seven chemical classes (Tale 2).The major class was constituted by the oxygenated monoterpenes $(46.5 \pm 0.0-85.6 \pm 0.0)$, with 1,8 -cineole (39.1 $\pm 0.0-79.4 \pm 0.0 \%)$ having the highest content in all the studies species, whatever their provenance. It was followed by $\alpha$-terpineol $(0.2 \pm 0.0-8.8 \pm 1.1 \%)$ and $\alpha$-terpinyl acetate $(0.0-$ $2.6 \pm 0.6 \%)$. The class with the second highest contents composed of the monoterpenes hydrocarbons $(4.5 \pm 0.0-$ $35.0 \pm 0.0 \%)$ represented by $\alpha$-pinene $(2.5 \pm 0.0-30.0 \pm 0.0 \%)$, limonene $(1.0 \pm 0.0-4.3 \pm 0.8 \%), \quad p$-cymene $(0.7 \pm 0.0-$ $2.7 \pm 2.1 \%$ ) and $g$-terpinene (traces to $0.3 \pm 0.0 \%$ ). The class with the third highest contents was composed by the oxygenated sesquiterpenes $(1.4 \pm 0.4-12.8 \pm 0.0 \%)$, constituted essentially by globulol $(0.3 \pm 0.0-5.7 \pm 1.3 \%)$, viridiflorol $(0.2 \pm 0.0-$ $1.8 \pm 0.0 \%$ ), epiglobulol (traces to $1.3 \pm 0.6 \%$ ) and spathulenol $(0 . \pm 0.0-1.3 \pm 0.0 \%)$. The sesquiterpenes hydrocarbons occupied the fourth position with a mean percentage varying between $1.4 \pm 0.1$ and $8.0 \pm 2.1 \%$, from wich the aromadendrene $(0.1 \pm 0.0-4.9 \pm 2.1 \%)$ was the major compound, followed by bicyclogermacrene (traces to $3.1 \pm 0.0 \%$ ), $\beta$-carophyllene $(0.3 \pm 0.0-1.9 \pm 0.0 \%)$ and allo-aromadendrene $(0.1 \pm 0.0-$ $0.9 \pm 0.0 \%$ ). The ketones, esters and others were minor compound classes, not discussed in further details. The comparative study of the essential oils chemical composition according to the species and their origin of harvest (arboreta), shows that E. sideroxylon oils obtained from leaves harvested from Korbous arboreta are richer in oxygenated monoterpenes $(85.6 \pm 0.0 \%)$ and in 1,8 -cineole $(79.4 \pm 0.0)$, while it was it was characterized by the lowest content in $\alpha$-pinene (2.1 \pm 0.0$). E$. cinerea from Souiniat arboreta was also characterised by a relatively high mean percentage of 1,8-cineole $(71.8 \pm 0.0)$ against $69.8 \pm 2.2 \%$ and $70.7 \pm 1.5 \%$ for those from Souiniat and Zerniza arboreta; respectively, which were distinguished by their highest mean percentages of $\alpha$-terpineol $(8.8 \pm 1.1$, $7.5 \pm 0.6 \%$; respectively), limonene $(4.3 \pm 0.8, \quad 3.8 \pm 0.0 \%$; respectively) and $\alpha$-terpinyl acetate $(2.6 \pm 0.6,2.1 \pm 0.6 \%$; respectively). Oils of all the studied population of $E$. astringens oils have the lowest mean percentage in oxygenated monoterpenes $(16.5 \pm 1.9-35.0 \pm 0.0 \%)$ and in 1,8-cineole $(39.1 \pm 0.0-47.6 \pm 1.2 \%)$, however they were characterized by the highest mean percentage in $\alpha$-pinene, tr-pinocarveol, pinocarvone and attend the maximum in that of Korbous, Mrifek and Sejnene arboreta; respectively It was also observed that all the provenance of E. astringens are relatively rich in sesquipterpenes hydrocarbons $(6.8 \pm 0.3-8.0 \pm 2.1 \%)$, aromadendrene, allo-aromadendrene and bicyclogermacrene and in oxygenated sesquiterpens $(9.7 \pm 0.0-11.1 \pm 0.1 \%)$ represented essentially by gobulol, epiglobulol and guaiol. 
Table2. Chemical Composition of the Essential Oils Extracted from the Leafs of 3 Eucalyptus Species harvested from six arboreta

\begin{tabular}{|c|c|c|c|c|c|c|c|c|c|c|c|c|}
\hline \multirow[t]{3}{*}{ Compounds and names } & \multirow[t]{3}{*}{ KI } & \multicolumn{11}{|c|}{ Composition (\%) } \\
\hline & & \multicolumn{3}{|c|}{ E. cinerea } & \multicolumn{3}{|c|}{ E. astringens } & \multicolumn{5}{|c|}{ E. sideroxylon } \\
\hline & & 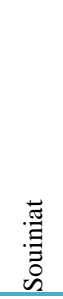 & $\begin{array}{l}\stackrel{\mathbb{N}}{E} \\
\stackrel{\mathbb{N}}{N}\end{array}$ & 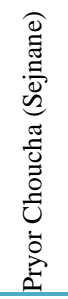 & 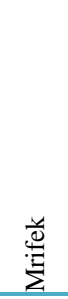 & 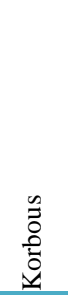 & 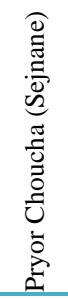 & 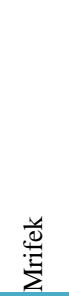 & 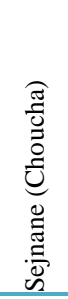 & 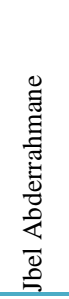 & $\begin{array}{l}\mathscr{z} \\
0 \\
0 \\
0 \\
0\end{array}$ & $\begin{array}{l}\stackrel{\vec{J}}{\Xi} \\
\stackrel{\Xi}{\Xi} \\
\mathscr{n}\end{array}$ \\
\hline Monoterpene hydrocarbons & & 11.1 & 10.2 & 10.3 & 24.4 & 35 & 16.5 & 15.4 & 8.2 & 4.5 & 4.9 & 10.5 \\
\hline Tricyclene & 1043 & - & $\operatorname{tr}$ & $\operatorname{tr}$ & - & - & - & - & $\operatorname{Tr}$ & - & - & - \\
\hline$\alpha$-Pinene & 1050 & 5.1 & 5.2 & 5.7 & 21.8 & 30.0 & 14 & 8.3 & 2.9 & 2.5 & 2,1 & 5,1 \\
\hline$\alpha$-Thyjene & 1053 & $\operatorname{tr}$ & $\operatorname{tr}$ & $\operatorname{tr}$ & $\operatorname{tr}$ & 0.1 & $\operatorname{tr}$ & 0.2 & 0.1 & - & $\operatorname{tr}$ & $\operatorname{tr}$ \\
\hline$\alpha$-Fenchene & 1072 & $\operatorname{tr}$ & $\operatorname{tr}$ & $\operatorname{tr}$ & 0.1 & $\operatorname{tr}$ & $\operatorname{tr}$ & $\operatorname{tr}$ & $\operatorname{tr}$ & $\operatorname{tr}$ & $\operatorname{tr}$ & $\operatorname{tr}$ \\
\hline Camphene & 1077 & 0.1 & 0.1 & 0.1 & 0.2 & 0.1 & 0.1 & 0.1 & 0.1 & tr & 0,1 & 0,1 \\
\hline$\alpha$-Pinene & 1108 & 0.1 & $\operatorname{tr}$ & 0.1 & 0.2 & 0.5 & 0.2 & 0.2 & 0.1 & 0.1 & 0,1 & 0,2 \\
\hline Sabinene & 1125 & - & - & - & - & - & - & $\operatorname{tr}$ & - & - & - & $\operatorname{tr}$ \\
\hline Verbenene & 1127 & - & - & - & 0.1 & $\operatorname{tr}$ & 0.1 & $\operatorname{tr}$ & - & - & $\operatorname{tr}$ & $\operatorname{tr}$ \\
\hline 2.4(10)-Tujadiene & 1132 & - & - & - & - & - & - & $\operatorname{tr}$ & - & - & - & - \\
\hline$\sigma$-3-Carene & 1146 & - & - & - & - & - & - & - & $\operatorname{tr}$ & - & - & - \\
\hline$\alpha$-Phellandrene & 1168 & 0.1 & 0.1 & 0.1 & 0.1 & 0.4 & 0.1 & 1.3 & 0.2 & 0.1 & $\operatorname{tr}$ & 0,4 \\
\hline Myrcene & 1172 & $\operatorname{tr}$ & $\operatorname{tr}$ & $\operatorname{tr}$ & $\operatorname{tr}$ & - & - & $\operatorname{tr}$ & $\operatorname{tr}$ & - & $\operatorname{tr}$ & $\operatorname{tr}$ \\
\hline$\alpha$-Terpinene & 1183 & - & - & - & - & - & - & 0.1 & - & - & - & - \\
\hline Limonene & 1207 & 4.3 & 3.8 & 3.3 & 1 & 1.9 & 0.6 & 3.9 & 1.7 & 1 & 1,5 & 3,3 \\
\hline$\alpha$-Phellandrene & 1209 & - & - & - & - & - & - & - & - & - & - & 0,3 \\
\hline$\alpha$-cis-Ocimene & 1240 & $\operatorname{tr}$ & $\operatorname{tr}$ & - & - & tr & - & $\operatorname{tr}$ & $\operatorname{tr}$ & - & $\operatorname{tr}$ & $\operatorname{tr}$ \\
\hline$\alpha$-Terpinene & 1251 & 0.1 & 0.1 & $\operatorname{tr}$ & $\operatorname{tr}$ & tr & $\operatorname{tr}$ & 0.3 & 0.3 & $\operatorname{tr}$ & 0,1 & 0,1 \\
\hline$\alpha$-trans-Ocimene & 1256 & $\operatorname{tr}$ & $\operatorname{tr}$ & 0.1 & $\operatorname{tr}$ & $\operatorname{tr}$ & $\operatorname{tr}$ & $\operatorname{tr}$ & 0.1 & 0.1 & 0,1 & $\operatorname{tr}$ \\
\hline$p$-Cymene & 1278 & 1 & 0.7 & 0.7 & 0.8 & 1.7 & 1.3 & 0.9 & 2.7 & 0.7 & 0,8 & 0,8 \\
\hline Terpinolene & 1289 & 0.1 & 0.1 & 0.1 & $\operatorname{tr}$ & 0.1 & $\operatorname{tr}$ & 0.1 & $\operatorname{tr}$ & - & $\operatorname{tr}$ & 0,1 \\
\hline Oxygenated monoterpenes & & 85 & 84.9 & 85 & 56.4 & 46.5 & 64 & 75.1 & 77.8 & 70.4 & 85.6 & 80.2 \\
\hline$p$ - $\alpha$-Dimethyl styrene & 1449 & 0.1 & 0.1 & 0.1 & $\operatorname{tr}$ & tr & $\operatorname{tr}$ & $\operatorname{tr}$ & $\operatorname{tr}$ & $\operatorname{tr}$ & $\operatorname{tr}$ & $\operatorname{tr}$ \\
\hline 1.8-Cineole & 1218 & 69.8 & 70.7 & 71.8 & 40.1 & 39.1 & 47.6 & 66.7 & 71.2 & 64.1 & 79,4 & 72,3 \\
\hline$p$-Mentha-6.8-dien-2-ol & 1222 & $\operatorname{tr}$ & $\operatorname{tr}$ & $\operatorname{tr}$ & $\operatorname{tr}$ & - & - & $\operatorname{tr}$ & $\operatorname{tr}$ & - & $\operatorname{tr}$ & $\operatorname{tr}$ \\
\hline trans-Rose oxyde & 1366 & - & - & - & 0.1 & - & 0.1 & - & - & - & - & - \\
\hline$\alpha-\alpha$-Pinene epoxyde & 1384 & $\operatorname{tr}$ & $\operatorname{tr}$ & - & $\operatorname{tr}$ & - & - & - & $\operatorname{tr}$ & - & - & $\operatorname{tr}$ \\
\hline$\alpha$-Thujone & 1423 & 0.1 & 0.1 & 0.1 & $\operatorname{tr}$ & - & $\operatorname{tr}$ & tr & $\operatorname{tr}$ & - & $\operatorname{tr}$ & tr \\
\hline cis-Linalool oxyde & 1455 & $\operatorname{tr}$ & - & - & - & - & - & - & - & - & - & $\operatorname{tr}$ \\
\hline trans-Linalool oxyde & 1468 & $\operatorname{tr}$ & $\operatorname{tr}$ & $\operatorname{tr}$ & - & - & - & $\operatorname{tr}$ & - & - & - & - \\
\hline Citronellal & 1491 & - & - & - & - & - & - & $\operatorname{tr}$ & - & - & - & - \\
\hline$\alpha$-Campholenic aldehyde & 1502 & $\operatorname{tr}$ & $\operatorname{tr}$ & $\operatorname{tr}$ & 0.1 & 0.1 & 0.1 & $\operatorname{tr}$ & 0.1 & $\operatorname{tr}$ & $\operatorname{tr}$ & $\operatorname{tr}$ \\
\hline Camphor & 1531 & $\operatorname{tr}$ & $\operatorname{tr}$ & $\operatorname{tr}$ & - & - & - & - & $\operatorname{tr}$ & - & $\operatorname{tr}$ & $\operatorname{tr}$ \\
\hline iso-Pinocamphone & 1533 & - & - & - & 0.1 & $\operatorname{tr}$ & 0.1 & - & - & - & - & - \\
\hline Linalool & 1560 & $\operatorname{tr}$ & - & - & $\operatorname{tr}$ & $\operatorname{tr}$ & $\operatorname{tr}$ & $\operatorname{tr}$ & $\operatorname{tr}$ & - & $\operatorname{tr}$ & - \\
\hline trans-p-Menth-2-en-1-ol & 1572 & $\operatorname{tr}$ & - & $\operatorname{tr}$ & - & - & - & $\operatorname{tr}$ & $\operatorname{tr}$ & - & - & $\operatorname{tr}$ \\
\hline
\end{tabular}




\begin{tabular}{|c|c|c|c|c|c|c|c|c|c|c|c|c|}
\hline cis-Sabinene hydrate & 1576 & - & - & - & - & - & - & - & $\operatorname{tr}$ & - & $\operatorname{tr}$ & - \\
\hline Isopulegol & 1581 & - & - & - & $\operatorname{tr}$ & $\operatorname{tr}$ & $\operatorname{tr}$ & - & $\operatorname{tr}$ & - & - & - \\
\hline Pinocarvone & 1585 & 0.2 & 0.3 & 0.4 & 2.9 & 0.8 & 3.7 & 0.2 & 0.3 & 0.4 & 0,3 & 0,2 \\
\hline Fenchol & 1593 & 0.1 & 0.2 & 0.1 & 0.3 & 0.2 & 0.3 & 0.1 & 0.1 & 0.1 & 0,1 & 0,1 \\
\hline Terpinene-4-ol & 1618 & $\operatorname{tr}$ & $\operatorname{tr}$ & $\operatorname{tr}$ & 0.2 & 0.2 & 0.1 & 0.2 & 0.1 & 0.2 & 0,1 & 0,1 \\
\hline cis-p-Menth-2-en-1-ol & 1634 & - & - & - & - & - & - & - & - & - & - & - \\
\hline Myrtenal & 1638 & $\operatorname{tr}$ & $\operatorname{tr}$ & 0.1 & $\operatorname{tr}$ & $\operatorname{tr}$ & $\operatorname{tr}$ & 0.1 & 0.1 & 0.1 & 0,1 & 0,1 \\
\hline Umbellulone & 1655 & - & - & - & $\operatorname{tr}$ & $\operatorname{tr}$ & - & - & - & - & - & - \\
\hline trans-pinocarveol & 1670 & 0.7 & 0.9 & 0.9 & 10.0 & 3.7 & 9.3 & 1 & 1.3 & 1.8 & 1,5 & 1,1 \\
\hline trans-p-Menth-2-ene-1.8-diol & 1673 & - & - & - & - & - & - & $\operatorname{tr}$ & $\operatorname{tr}$ & - & - & $\operatorname{tr}$ \\
\hline$\sigma$-Terpineol & 1686 & 0.1 & $\operatorname{tr}$ & - & $\operatorname{tr}$ & 0.1 & 0.1 & 0.1 & 0.1 & 0.2 & - & 0,1 \\
\hline Cis-Piperitol & 1698 & $\operatorname{tr}$ & - & - & $\operatorname{tr}$ & $\operatorname{tr}$ & $\operatorname{tr}$ & - & $\operatorname{tr}$ & - & $\operatorname{tr}$ & $\operatorname{tr}$ \\
\hline CarvotanAcetone & 1699 & $\operatorname{tr}$ & - & - & - & - & - & 0.1 & - & - & - & - \\
\hline Neral & 1705 & - & - & - & - & - & - & $\operatorname{tr}$ & - & - & - & - \\
\hline$\alpha$-Terpineol & 1708 & 8.8 & 7.5 & 6.4 & 0.7 & 0.9 & 0.5 & 2.1 & 0.8 & 0.5 & 0,2 & 1,8 \\
\hline$\alpha$-Terpinyl acetate & 1708 & 2.6 & 2.1 & 2 & $\operatorname{tr}$ & - & - & 2.9 & 0.9 & 0.7 & 0,8 & 2,8 \\
\hline Borneol & 1710 & 0.2 & 0.3 & 0.2 & 0.2 & 0.3 & 0.1 & 0.2 & 0.1 & 0.1 & 0,1 & 0,2 \\
\hline Phellandral & 1740 & - & - & - & - & - & - & - & - & - & - & - \\
\hline Piperitone & 1746 & 0.1 & - & - & $\operatorname{tr}$ & $\operatorname{tr}$ & $\operatorname{tr}$ & $\operatorname{tr}$ & - & - & - & $\operatorname{tr}$ \\
\hline Carvone & 1754 & 0.2 & 0.2 & 0.2 & - & - & - & - & 0.1 & - & 0,1 & - \\
\hline Geranial & 1757 & - & - & - & - & - & - & $\operatorname{tr}$ & - & - & - & - \\
\hline Geranial & 1761 & tr & - & - & $\operatorname{tr}$ & - & $\operatorname{tr}$ & $\operatorname{tr}$ & $\operatorname{tr}$ & - & $\operatorname{tr}$ & $\operatorname{tr}$ \\
\hline Geranyl acetate & 1763 & tr & - & $\operatorname{tr}$ & - & - & - & $\operatorname{tr}$ & - & $\operatorname{tr}$ & - & - \\
\hline trans-Piperitol & 1767 & $\operatorname{tr}$ & - & - & $\operatorname{tr}$ & - & $\operatorname{tr}$ & $\operatorname{tr}$ & 0.1 & 0.1 & 0,1 & $\operatorname{tr}$ \\
\hline Citroneoll & 1785 & $\operatorname{tr}$ & - & - & - & - & - & - & - & - & - & - \\
\hline Cuminal & 1801 & $\operatorname{tr}$ & - & - & - & - & $\operatorname{tr}$ & $\operatorname{tr}$ & $\operatorname{tr}$ & - & - & $\operatorname{tr}$ \\
\hline Cuminal & 1803 & - & - & - & - & - & - & - & - & - & - & - \\
\hline Myrtenol & 1807 & $\operatorname{tr}$ & $\operatorname{tr}$ & $\operatorname{tr}$ & 0.2 & 0.1 & 0.2 & 0.1 & 0.1 & 0.1 & 0,1 & $\operatorname{tr}$ \\
\hline trans-p-Mentha-1(7).8dien-2-ol & 1811 & 0.7 & 0.8 & 0.9 & 0.4 & 0.2 & 0.4 & 0.2 & 0.8 & 0.8 & 1 & 0,4 \\
\hline Nerol & 1818 & $\operatorname{tr}$ & $\operatorname{tr}$ & $\operatorname{tr}$ & $\operatorname{tr}$ & 0.1 & $\operatorname{tr}$ & $\operatorname{tr}$ & $\operatorname{tr}$ & $\operatorname{tr}$ & $\operatorname{tr}$ & $\operatorname{tr}$ \\
\hline$\alpha$-Phellandrene epoxyde & 1822 & $\operatorname{tr}$ & - & - & $\operatorname{tr}$ & $\operatorname{tr}$ & $\operatorname{tr}$ & $\operatorname{tr}$ & $\operatorname{tr}$ & - & - & $\operatorname{tr}$ \\
\hline trans-Carveol & 1848 & 0.2 & 0.2 & 0.1 & 0.2 & 0.1 & 0.2 & 0.1 & 0.2 & 0.1 & 0,2 & 0,1 \\
\hline trans-p-Menth-1.8-dien-6-ol & 1856 & $\operatorname{tr}$ & $\operatorname{tr}$ & $\operatorname{tr}$ & $\operatorname{tr}$ & $\operatorname{tr}$ & - & $\operatorname{tr}$ & - & - & - & $\operatorname{tr}$ \\
\hline$p$-Cymen-8-ol & 1863 & 0.1 & 0.1 & 0.2 & 0.1 & 0.1 & 0.1 & 0.1 & 0.1 & 0.1 & 0,1 & 0,1 \\
\hline Geraniol & 1867 & $\operatorname{tr}$ & - & - & - & - & - & - & - & - & - & $\operatorname{tr}$ \\
\hline Neryl acetone & 1875 & - & - & - & $\operatorname{tr}$ & $\operatorname{tr}$ & - & - & - & - & - & - \\
\hline cis-Carveol & 1879 & 0.1 & 0.2 & 0.2 & $\operatorname{tr}$ & $\operatorname{tr}$ & $\operatorname{tr}$ & $\operatorname{tr}$ & 0.1 & $\operatorname{tr}$ & 0,1 & $\operatorname{tr}$ \\
\hline cis-p-Mentha-1.8-dien-6-ol & 1884 & $\operatorname{tr}$ & - & - & - & - & - & - & - & - & - & - \\
\hline cis-p-Mentha-1(7).8dien-2-ol & 1903 & 0.7 & 0.9 & 0.9 & 0.3 & 0.2 & 0.4 & 0.3 & 0.8 & 0.8 & 0,9 & 0,4 \\
\hline p-Mentha-1.5-dien-7-o & 1984 & - & - & - & - & - & - & - & - & - & - & - \\
\hline Methyl eugenol & 2026 & - & - & - & $\operatorname{tr}$ & $\operatorname{tr}$ & 0.1 & $\operatorname{tr}$ & $\operatorname{tr}$ & $\operatorname{tr}$ & $\operatorname{tr}$ & $\operatorname{tr}$ \\
\hline$p$-Cymen7-ol & 2121 & - & - & - & $\operatorname{tr}$ & - & $\operatorname{tr}$ & - & $\operatorname{tr}$ & - & $\operatorname{tr}$ & $\operatorname{tr}$ \\
\hline Thymol & 2172 & $\operatorname{tr}$ & $\operatorname{tr}$ & $\operatorname{tr}$ & 0.1 & $\operatorname{tr}$ & 0.1 & - & 0.1 & tr & $\operatorname{tr}$ & 0,1 \\
\hline Eugenol & 2191 & $\operatorname{tr}$ & $\operatorname{tr}$ & $\operatorname{tr}$ & - & - & - & - & - & - & - & - \\
\hline Carvacrol & 2233 & 0.1 & 0.1 & 0.1 & 0.1 & 0.2 & 0.2 & 0.3 & 0.2 & - & 0,1 & $\operatorname{tr}$ \\
\hline Carvacrol & 2239 & - & - & - & 0.1 & - & $\operatorname{tr}$ & - & - & - & - & $\operatorname{tr}$ \\
\hline
\end{tabular}




\begin{tabular}{|c|c|c|c|c|c|c|c|c|c|c|c|c|}
\hline$p$-Cuminol & 2240 & - & - & - & - & - & - & - & - & - & - & - \\
\hline 1-Terpineol & 1643 & - & - & $\operatorname{tr}$ & - & - & 0.1 & - & $\operatorname{tr}$ & $\operatorname{tr}$ & $\operatorname{tr}$ & - \\
\hline 1.8-Menthadien-4-ol & 1701 & 0.1 & 0.1 & 0.2 & - & - & 0.1 & 0.1 & 0.1 & 0.1 & $\operatorname{tr}$ & $\operatorname{tr}$ \\
\hline Sesquiterpene hydrocarbons & & 1.6 & 1.8 & 1.4 & 8.0 & 7.7 & 6.8 & 4.8 & 4.8 & 10.5 & 3.2 & 4.1 \\
\hline Isoledene & 1477 & - & 0.1 & 0.1 & $\operatorname{tr}$ & 0.1 & $\operatorname{tr}$ & $\operatorname{tr}$ & $\operatorname{tr}$ & 0.1 & $\operatorname{tr}$ & 0,1 \\
\hline$\alpha$-Cubebene & 1479 & 0.1 & - & - & $\operatorname{tr}$ & - & - & $\operatorname{tr}$ & - & - & - & - \\
\hline Bicycloelemene & 1493 & - & - & - & $\operatorname{tr}$ & $\operatorname{tr}$ & - & $\operatorname{tr}$ & - & - & - & - \\
\hline$\alpha$-Copaene & 1506 & - & $\operatorname{tr}$ & - & - & - & - & - & $\operatorname{tr}$ & - & - & - \\
\hline$\alpha$-Gurjunene & 1542 & tr & $\operatorname{tr}$ & $\operatorname{tr}$ & $\operatorname{tr}$ & 0.1 & $\operatorname{tr}$ & 0.1 & $\operatorname{tr}$ & 0.1 & $\operatorname{tr}$ & $\operatorname{tr}$ \\
\hline$\beta$-Cubebene & 1554 & - & $\operatorname{tr}$ & $\operatorname{tr}$ & $\operatorname{tr}$ & $\operatorname{tr}$ & - & $\operatorname{tr}$ & $\operatorname{tr}$ & - & - & $\operatorname{tr}$ \\
\hline$\beta$-Elemene & 1602 & - & - & - & $\operatorname{tr}$ & $\operatorname{tr}$ & - & $\operatorname{tr}$ & $\operatorname{tr}$ & 0.1 & $\operatorname{tr}$ & $\operatorname{tr}$ \\
\hline$\beta$-Gurjunene & 1607 & - & - & - & 0.2 & 0.1 & 0.2 & $\operatorname{tr}$ & 0.1 & 0.1 & 0,1 & $\operatorname{tr}$ \\
\hline$\beta$-Caryophyllene & 1613 & 0.7 & 0.6 & 0.6 & 0.3 & 1 & 1 & 1.4 & 0.8 & 1.9 & 0,4 & 1 \\
\hline$\alpha$-Guaiene & 1619 & - & - & - & - & - & - & $\operatorname{tr}$ & - & - & - & - \\
\hline Aromadendrene & 1622 & 0.2 & 0.2 & 0.1 & 4.9 & 3.8 & 3.8 & 0.4 & 1.8 & 3.2 & 1,2 & 0,5 \\
\hline Dehydraromadendrene & 1630 & $\operatorname{tr}$ & $\operatorname{tr}$ & $\operatorname{tr}$ & 0.1 & 0.1 & 0.1 & 0.1 & 0.1 & 0.1 & 0,1 & 0,1 \\
\hline Alooaromadendrene & 1661 & 0.1 & 0.2 & 0.1 & 0.6 & 0.6 & 0.6 & 0.2 & 0.5 & 0.9 & 0,4 & 0,2 \\
\hline$\alpha$-Humulene & 1683 & 0.2 & 0.3 & 0.2 & 0.1 & 0.1 & 0.1 & 0.2 & 0.2 & 0.2 & 0,2 & 0,3 \\
\hline Ledene & 1703 & $\operatorname{tr}$ & $\operatorname{tr}$ & $\operatorname{tr}$ & $\operatorname{tr}$ & $\operatorname{tr}$ & - & $\operatorname{tr}$ & - & - & - & - \\
\hline Germacrene D & 1712 & - & - & - & - & - & - & - & - & - & - & - \\
\hline$\beta$-Selinene & 1715 & $\operatorname{tr}$ & - & - & 0.5 & 0.2 & 0.4 & - & - & - & - & - \\
\hline$\alpha-$ Selinene & 1727 & - & - & $\operatorname{tr}$ & $\operatorname{tr}$ & $\operatorname{tr}$ & 0.1 & 0.1 & $\operatorname{tr}$ & 0.1 & $\operatorname{tr}$ & 0,1 \\
\hline A-Muurolene & 1740 & $\operatorname{tr}$ & $\operatorname{tr}$ & $\operatorname{tr}$ & 0.4 & 0.2 & - & 0.2 & $\operatorname{tr}$ & - & $\operatorname{tr}$ & 0,3 \\
\hline Bicyclogermacrene & 1754 & $\operatorname{tr}$ & - & - & 0.2 & 0.5 & 0.1 & 1.6 & 0.6 & 3.1 & 0,4 & 1,1 \\
\hline$\sigma$-Cadinene & 1768 & $\operatorname{tr}$ & $\operatorname{tr}$ & 0.1 & $\operatorname{tr}$ & $\operatorname{tr}$ & $\operatorname{tr}$ & 0.1 & 0.1 & $\operatorname{tr}$ & $\operatorname{tr}$ & 0,1 \\
\hline$\sigma$-Cadinene & 1773 & $\operatorname{tr}$ & $\operatorname{tr}$ & - & $\operatorname{tr}$ & $\operatorname{tr}$ & $\operatorname{tr}$ & $\operatorname{tr}$ & $\operatorname{tr}$ & - & $\operatorname{tr}$ & 0,1 \\
\hline$\gamma$-Cadinene & 1777 & $\operatorname{tr}$ & - & - & $\operatorname{tr}$ & $\operatorname{tr}$ & $\operatorname{tr}$ & $\operatorname{tr}$ & $\operatorname{tr}$ & $\operatorname{tr}$ & - & $\operatorname{tr}$ \\
\hline cis-Calamenene & 1839 & $\operatorname{tr}$ & 0.1 & 0.1 & - & - & - & $\operatorname{tr}$ & $\operatorname{tr}$ & - & $\operatorname{tr}$ & $\operatorname{tr}$ \\
\hline$\alpha$-Calacorene & 1938 & - & - & - & - & - & - & $\operatorname{tr}$ & $\operatorname{tr}$ & - & $\operatorname{tr}$ & $\operatorname{tr}$ \\
\hline Eremophilene & 2137 & 0.1 & 0.2 & 0.1 & 0.5 & 0.6 & 0.4 & 0.2 & 0.3 & 0.7 & 0,3 & 0,2 \\
\hline$\beta$-Maaliene & 2162 & - & - & - & $\operatorname{tr}$ & $\operatorname{tr}$ & $\operatorname{tr}$ & - & $\operatorname{tr}$ & $\operatorname{tr}$ & - & - \\
\hline$\alpha$-Amorphene & 1690 & - & - & - & $\operatorname{tr}$ & - & - & - & - & - & - & - \\
\hline Oxygenated Sesquiterpenes & & 1.6 & 2.2 & 1.4 & 10.1 & 9.7 & 11.1 & 3.6 & 7.6 & 12.8 & 5 & 4.1 \\
\hline Palustrol & 1950 & $\operatorname{tr}$ & 0.1 & $\operatorname{tr}$ & 0.1 & 0.1 & 0.1 & 0.1 & 0.1 & 0.2 & 0,1 & 0,1 \\
\hline Caryophyllene oxyde & 2010 & 0.1 & 0.1 & 0.1 & $\operatorname{tr}$ & 0.1 & 0.4 & 0.2 & 0.2 & 0.3 & 0,2 & 0,1 \\
\hline Epiglobulol & 2033 & 0.1 & 0.1 & $\operatorname{tr}$ & 1.3 & 0.9 & 1.1 & 0.1 & 0.5 & 0.7 & 0,3 & 0,1 \\
\hline Ledol & 2053 & tr & 0.1 & $\operatorname{tr}$ & 0.2 & 0.2 & 0.2 & 0.1 & 0.2 & 0.3 & 0,1 & 0,1 \\
\hline trans-Nerolidol & 2068 & $\operatorname{tr}$ & - & - & $\operatorname{tr}$ & $\operatorname{tr}$ & 0.1 & - & $\operatorname{tr}$ & $\operatorname{tr}$ & $\operatorname{tr}$ & $\operatorname{tr}$ \\
\hline Elemol & 2075 & 0.1 & 0.2 & 0.1 & 0.4 & 0.4 & 0.3 & 0.2 & 0.3 & 0.6 & 0,2 & 0,2 \\
\hline$\beta$-Oplopenone & 2087 & - & - & - & $\operatorname{tr}$ & $\operatorname{tr}$ & $\operatorname{tr}$ & 0.1 & 0.1 & 0.1 & $\operatorname{tr}$ & 0,1 \\
\hline Globulol & 2097 & 0.5 & 0.6 & 0.3 & 5.7 & 4.5 & 5.3 & 0.9 & 3 & 4.7 & 2,1 & 1 \\
\hline Viridiflorol & 2107 & 0.2 & 0.4 & 0.2 & 0.9 & 1 & 0.9 & 0.5 & 0.7 & 1.8 & 0,5 & 0,5 \\
\hline Guaiol & 2129 & 0.1 & 0.1 & 0.1 & 0.5 & 0.5 & 0.5 & 0.2 & 0.3 & 0.6 & 0,2 & 0,2 \\
\hline Rosifoliol & 2130 & 0.1 & $\operatorname{tr}$ & - & - & - & - & - & - & - & - & - \\
\hline Spathulenol & 2145 & 0.2 & 0.3 & 0.2 & 0.4 & 1.1 & 1.1 & 0.5 & 0.8 & 1.3 & 0,5 & 0,6 \\
\hline$T$-Cadinol & 2166 & - & - & - & $\operatorname{tr}$ & $\operatorname{tr}$ & 0.1 & 0.1 & $\operatorname{tr}$ & 0.2 & $\operatorname{tr}$ & 0,1 \\
\hline
\end{tabular}




\begin{tabular}{|c|c|c|c|c|c|c|c|c|c|c|c|c|}
\hline$T$-Muurolool & 2183 & $\operatorname{tr}$ & $\operatorname{tr}$ & $\operatorname{tr}$ & $\operatorname{tr}$ & tr & $\operatorname{tr}$ & $\operatorname{tr}$ & 0.1 & $\operatorname{tr}$ & $\operatorname{tr}$ & $\operatorname{tr}$ \\
\hline$\gamma$-Eudesmol & 2190 & - & - & - & $\operatorname{tr}$ & 0.1 & 0.1 & $\operatorname{tr}$ & 0.1 & 0.1 & 0,1 & 0,1 \\
\hline$\sigma$-Cadinol & 2201 & $\operatorname{tr}$ & $\operatorname{tr}$ & $\operatorname{tr}$ & $\operatorname{tr}$ & 0.1 & 0.1 & 0.1 & 0.1 & 0.1 & $\operatorname{tr}$ & 0,1 \\
\hline Agarospirol & 2208 & $\operatorname{tr}$ & $\operatorname{tr}$ & $\operatorname{tr}$ & $\operatorname{tr}$ & 0.1 & 0.1 & 0.1 & 0.1 & 0.1 & $\operatorname{tr}$ & 0,1 \\
\hline$\alpha$-Cadinol & 2220 & $\operatorname{tr}$ & $\operatorname{tr}$ & $\operatorname{tr}$ & $\operatorname{tr}$ & tr & $\operatorname{tr}$ & $\operatorname{tr}$ & 0.1 & tr & $\operatorname{tr}$ & $\operatorname{tr}$ \\
\hline$\alpha$-Eudesmol & 2247 & $\operatorname{tr}$ & 0.1 & $\operatorname{tr}$ & 0.1 & 0.3 & 0.2 & 0.2 & 0.2 & 0.3 & 0,1 & 0,4 \\
\hline Isospathulenol & 2253 & - & - & - & - & - & - & - & - & 1.1 & - & 0,3 \\
\hline$\beta$-Eudesmol & 2258 & 0.1 & 0.1 & 0.1 & 0.1 & 0.1 & 0.5 & 0.3 & 0.5 & - & 0,4 & 0,1 \\
\hline Farnesyl acetate & 2271 & - & - & - & tr & tr & $\operatorname{tr}$ & $\operatorname{tr}$ & 0.1 & - & - & $\operatorname{tr}$ \\
\hline Isobicyclogermacral & 2336 & $\operatorname{tr}$ & $\operatorname{tr}$ & $\operatorname{tr}$ & $\operatorname{tr}$ & $\operatorname{tr}$ & $\operatorname{tr}$ & 0.1 & $\operatorname{tr}$ & $\operatorname{tr}$ & - & 0,1 \\
\hline (E.E) Farnesol & 2374 & $\operatorname{tr}$ & $\operatorname{tr}$ & - & $\operatorname{tr}$ & - & 0.1 & $\operatorname{tr}$ & - & 0.2 & - & $\operatorname{tr}$ \\
\hline Esters & & 0.1 & 0.2 & 0.2 & 0.3 & 0.3 & 0.2 & 0.3 & 0.3 & 0.4 & 0.4 & 0.3 \\
\hline Isobutyl isobutyrate & 1091 & - & - & - & - & - & - & - & - & - & - & $\operatorname{tr}$ \\
\hline 4-Methyl-2- pentyl acetate & 1109 & - & - & - & $\operatorname{tr}$ & - & - & - & $\operatorname{tr}$ & - & - & $\operatorname{tr}$ \\
\hline Isoamyl acetate & 1126 & $\operatorname{tr}$ & $\operatorname{tr}$ & $\operatorname{tr}$ & - & - & - & - & $\operatorname{tr}$ & - & - & - \\
\hline Isoamyl isovalerate & 1300 & - & $\operatorname{tr}$ & 0.1 & - & - & - & 0.2 & 0.1 & $\operatorname{tr}$ & 0,2 & 0,2 \\
\hline Hexyl isobutyrate & 1334 & $\operatorname{tr}$ & 0.1 & $\operatorname{tr}$ & $\operatorname{tr}$ & - & $\operatorname{tr}$ & $\operatorname{tr}$ & $\operatorname{tr}$ & - & $\operatorname{tr}$ & $\operatorname{tr}$ \\
\hline Isoamyl valerate & 1410 & - & - & - & - & - & - & - & - & - & - & - \\
\hline Isoamyl hexanoate & 1466 & $\operatorname{tr}$ & - & - & - & - & - & - & $\operatorname{tr}$ & - & $\operatorname{tr}$ & - \\
\hline Amyl benzoate & 1939 & $\operatorname{tr}$ & $\operatorname{tr}$ & $\operatorname{tr}$ & $\operatorname{tr}$ & $\operatorname{tr}$ & $\operatorname{tr}$ & - & - & $\operatorname{tr}$ & - & - \\
\hline Benzyl pentanoate & 1956 & - & - & - & - & - & - & - & $\operatorname{tr}$ & - & - & - \\
\hline$\beta$-Phenylpropionate & 2020 & $\operatorname{tr}$ & 0.1 & $\operatorname{tr}$ & 0.2 & 0.2 & 0.2 & 0.1 & 0.2 & 0.3 & 0,2 & 0,1 \\
\hline Ketones & & 0.2 & 0.1 & 0.6 & 0.1 & 0.2 & 0.2 & 0.2 & 0.2 & 0.1 & 0.1 & 0.2 \\
\hline 6-Methyl-5-Hepten-2-one & 1345 & $\operatorname{tr}$ & - & - & $\operatorname{tr}$ & $\operatorname{tr}$ & - & $\operatorname{tr}$ & $\operatorname{tr}$ & - & $\operatorname{tr}$ & $\operatorname{tr}$ \\
\hline Nopinone & 1591 & - & - & - & - & - & - & - & - & - & - & - \\
\hline Cryptone & 1690 & $\operatorname{tr}$ & - & - & $\operatorname{tr}$ & $\operatorname{tr}$ & $\operatorname{tr}$ & 0.1 & 0.1 & tr & $\operatorname{tr}$ & $\operatorname{tr}$ \\
\hline 4-Methyl aceophenone & 1795 & $\operatorname{tr}$ & $\operatorname{tr}$ & 0.1 & $\operatorname{tr}$ & - & $\operatorname{tr}$ & $\operatorname{tr}$ & $\operatorname{tr}$ & - & $\operatorname{tr}$ & $\operatorname{tr}$ \\
\hline cis-Jasmone & 1964 & 0.1 & 0.1 & 0.1 & - & - & - & $\operatorname{tr}$ & $\operatorname{tr}$ & - & $\operatorname{tr}$ & $\operatorname{tr}$ \\
\hline Jacksone & 2289 & 0.1 & $\operatorname{tr}$ & 0.2 & $\operatorname{tr}$ & $\operatorname{tr}$ & - & $\operatorname{tr}$ & - & - & - & $\operatorname{tr}$ \\
\hline Tasmanone & 2305 & $\operatorname{tr}$ & $\operatorname{tr}$ & 0.2 & - & - & - & $\operatorname{tr}$ & - & - & - & - \\
\hline Agglomerone & 2390 & - & - & - & - & - & - & $\operatorname{tr}$ & - & - & - & 0,1 \\
\hline Lateriticone & 2394 & $\operatorname{tr}$ & $\operatorname{tr}$ & - & - & $\operatorname{tr}$ & 0.1 & - & - & - & - & - \\
\hline Torquatone & 2432 & - & - & - & 0.1 & 0.1 & 0.1 & - & $\operatorname{tr}$ & 0.1 & $\operatorname{tr}$ & - \\
\hline Others & & tr & tr & $\operatorname{tr}$ & tr & - & tr & $\operatorname{tr}$ & $\operatorname{tr}$ & - & tr & tr \\
\hline Hexanal & 1095 & $\operatorname{tr}$ & $\operatorname{tr}$ & $\operatorname{tr}$ & - & - & - & $\operatorname{tr}$ & - & - & - & $\operatorname{tr}$ \\
\hline trans-2-Hexenal & 1226 & $\operatorname{tr}$ & $\operatorname{tr}$ & $\operatorname{tr}$ & $\operatorname{tr}$ & - & $\operatorname{tr}$ & $\operatorname{tr}$ & $\operatorname{tr}$ & - & $\operatorname{tr}$ & $\operatorname{tr}$ \\
\hline Nonanal & 1401 & - & - & - & - & - & - & - & - & - & - & - \\
\hline Diphenyl oxyde & 2004 & - & - & - & $\operatorname{tr}$ & - & - & - & - & - & - & - \\
\hline cis-3-Hexenol & 1389 & - & - & - & $\operatorname{tr}$ & - & - & - & - & - & - & $\operatorname{tr}$ \\
\hline 2-Nonanol & 1523 & - & - & - & $\operatorname{tr}$ & - & - & $\operatorname{tr}$ & - & - & - & $\operatorname{tr}$ \\
\hline O-cresol & 1648 & - & - & - & - & - & - & - & - & - & - & - \\
\hline Toluene & 1063 & - & - & $\operatorname{tr}$ & - & - & - & - & - & - & - & - \\
\hline Total identified & & 99.5 & 99.4 & 98.8 & 99.3 & 99.2 & 98.8 & 99.4 & 99 & 98.8 & 99.2 & 99.4 \\
\hline
\end{tabular}




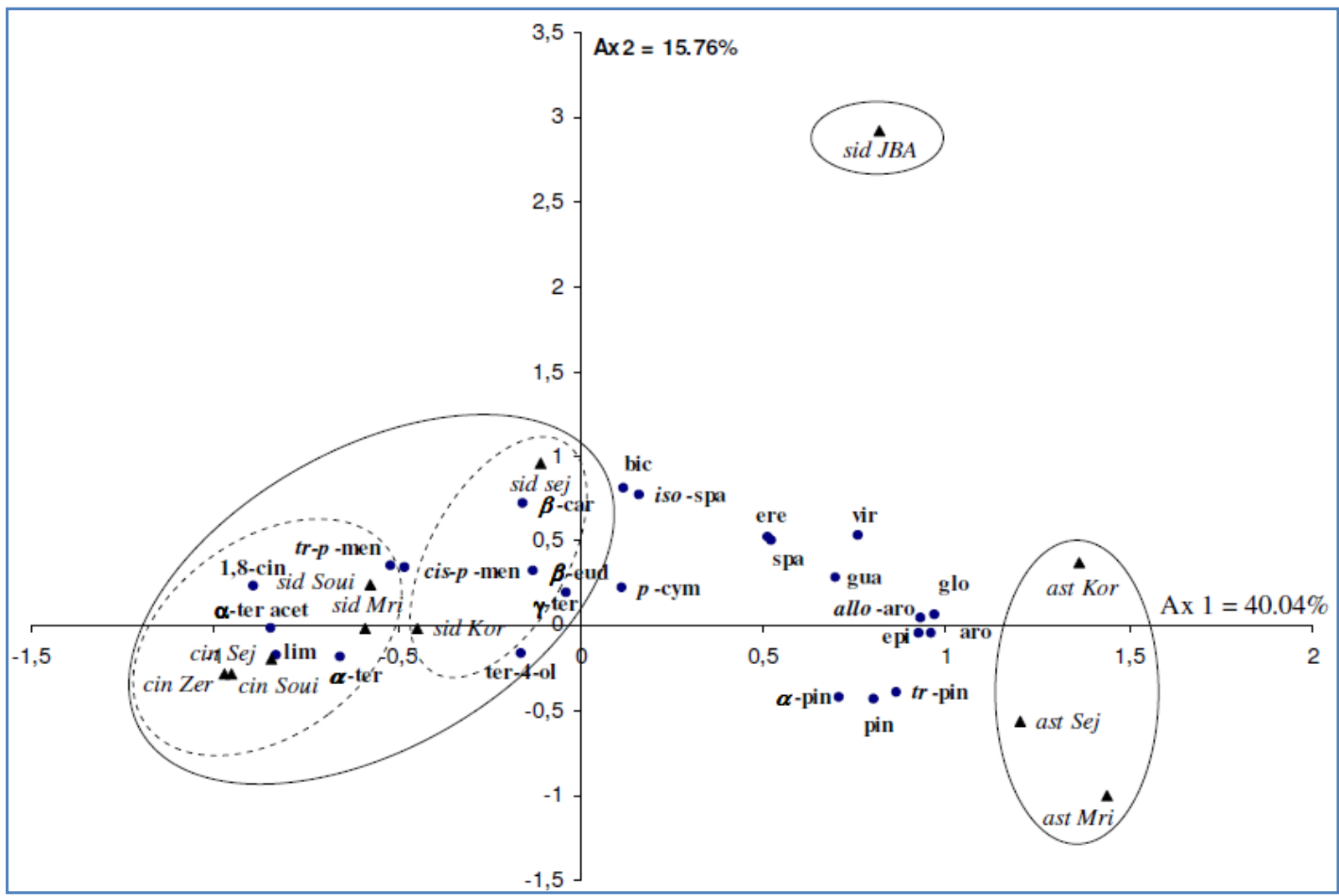

Figure 1 PCA of 24 compounds for the leaf essential oils of 3 Eucalyptus species. For the abbreviations of the Eucalyptus species ( $\triangle$ the arboreta and the compounds $(\bullet)$, refer Tables 1 and 3 for more detail.

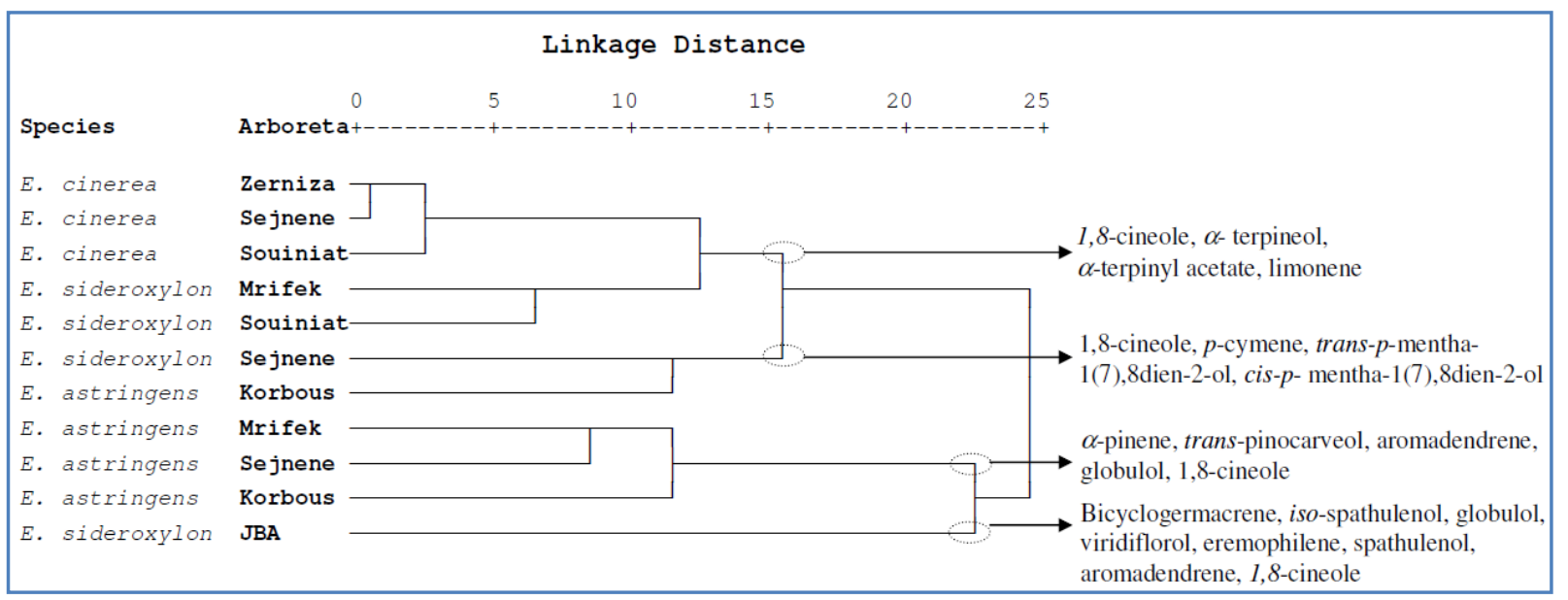

Figure 2 Dendrogram obtained by cluster analysis based on the Euclidean distances between groups of theleaf essential oils of 3 Tunisian

Eucalyptus species. Components that characterize the major subgroups, considered as chemotypes, are indicated. JBA = Jbel abderrahman 


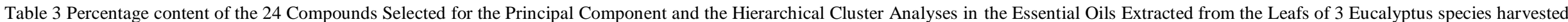
from six arboret

\begin{tabular}{|c|c|c|c|c|c|c|c|c|c|c|c|c|}
\hline \multirow[b]{2}{*}{ Compound } & \multirow[b]{2}{*}{$\begin{array}{l}\text { Arboreta } \\
\text { Abbreviation }\end{array}$} & \multicolumn{3}{|c|}{ E. cinerea } & \multicolumn{3}{|c|}{ E. astringens } & \multicolumn{5}{|c|}{ E. sideroxylon } \\
\hline & & 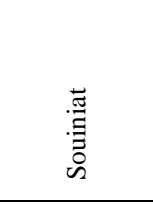 & 芯 & 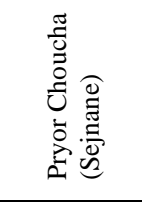 & 弟 & $\begin{array}{l}\tilde{0} \\
\stackrel{0}{0} \\
\stackrel{0}{u}\end{array}$ & 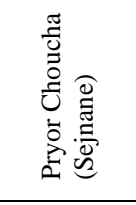 & $\stackrel{\frac{u}{0}}{\stackrel{D}{\Sigma}}$ & 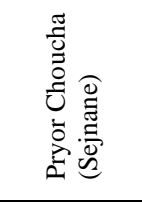 & 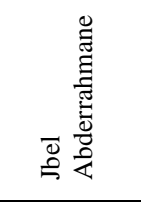 & $\begin{array}{l}\tilde{y} \\
\overline{0} \\
\overline{0} \\
\ddot{1}\end{array}$ & 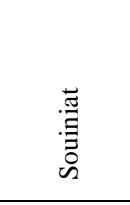 \\
\hline$\alpha$-Pinene & $\alpha$-pin & $5.1 \pm 0.8^{\mathrm{a}}$ & $5.2 \pm 0.6^{\mathrm{a}}$ & $5.7 \pm 1.4^{\mathrm{a}}$ & $21.8 \pm 4.7^{\mathrm{c}}$ & $30.0 \pm 0.0^{\mathrm{d}}$ & $14.0 \pm 1.7^{\mathrm{b}}$ & $8.3 \pm 6.4^{\mathrm{ab}}$ & $2.9 \pm 1.4^{\mathrm{a}}$ & $2.5 \pm 0.0^{\mathrm{a}}$ & $2.1 \pm 0.0^{\mathrm{a}}$ & $5.1 \pm 4.0^{\mathrm{a}}$ \\
\hline Limonene & $\lim$ & $4.3 \pm 0.8^{\mathrm{c}}$ & $3.8 \pm 0.0^{\mathrm{c}}$ & $3.3 \pm 0.0^{\mathrm{bc}}$ & $1.0 \pm 0.0^{\mathrm{a}}$ & $1.9 \pm 0.0^{\mathrm{ab}}$ & $0.6 \pm 0.0^{\mathrm{a}}$ & $3.9 \pm 0.5^{\mathrm{c}}$ & $1.7 \pm 0.7^{\mathrm{a}}$ & $1.0 \pm 0.0^{\mathrm{a}}$ & $1.5 \pm 0.0^{\mathrm{ab}}$ & $3.3 \pm 1.7^{\mathrm{c}}$ \\
\hline 1.8-Cineole & 1.8 -cin & $69.8 \pm 2.19^{c}$ & $70.7 \pm 1.5^{\mathrm{cd}}$ & $71.8 \pm 0.0^{\mathrm{cd}}$ & $40.1 \pm 5.79^{\mathrm{ab}}$ & $39.1 \pm 0.0^{\mathrm{a}}$ & $47.6 \pm 1.2^{\mathrm{b}}$ & $66.7 \pm 9.1^{\mathrm{c}}$ & $71.2 \pm 3.6^{\mathrm{cd}}$ & $64.2 \pm 0.0^{\mathrm{c}}$ & $79.4 \pm 0.0^{\mathrm{d}}$ & $72.3 \pm 2.7^{\mathrm{cd}}$ \\
\hline$\gamma$-Terpinene & $\gamma$-terp & $0.1 \pm 0.0^{\mathrm{a}}$ & $0.1 \pm 0.0^{\mathrm{a}}$ & $\operatorname{tr}^{\mathrm{a}}$ & $\operatorname{tr}^{\mathrm{a}}$ & $0.1 \pm 0.0^{\mathrm{a}}$ & $\operatorname{tr}^{\mathrm{a}}$ & $0.3 \pm 0.0^{\mathrm{a}}$ & $0.3 \pm 0.7^{\mathrm{b}}$ & $\operatorname{tr}^{\mathrm{a}}$ & $0.1 \pm 0.0^{\mathrm{a}}$ & $0.1 \pm 0.0^{\mathrm{a}}$ \\
\hline$p$-Cymene & -cym & $1.0 \pm 0.0^{\mathrm{a}}$ & $0.7 \pm 0.0^{\mathrm{a}}$ & $0.7 \pm 0.0^{\mathrm{a}}$ & $0.8 \pm 0.0^{\mathrm{a}}$ & $1.7 \pm 0.0^{\mathrm{b}}$ & $1.3 \pm 0.0^{\mathrm{a}}$ & $0.9 \pm 0.0^{\mathrm{a}}$ & $2.7 \pm 2.1^{\mathrm{b}}$ & $0.7 \pm 0.0^{\mathrm{a}}$ & $0.8 \pm 0.0^{\mathrm{a}}$ & $0.8 \pm 0.0^{\mathrm{a}}$ \\
\hline Pinocarvone & pin & $0.2 \pm 0.0^{\mathrm{a}}$ & $0.3 \pm 0.0^{\mathrm{a}}$ & $0.4 \pm 0.7^{\mathrm{ab}}$ & $2.9 \pm 0.84^{\mathrm{c}}$ & $0.8 \pm 0.0^{\mathrm{b}}$ & $3.7 \pm 0.6^{\mathrm{d}}$ & $0.2 \pm 0.0^{\mathrm{a}}$ & $0.3 \pm 0.0^{\mathrm{a}}$ & $0.4 \pm 0.0^{\mathrm{a}}$ & $0.4 \pm 0.0^{\mathrm{a}}$ & $0.2 \pm 0.0^{\mathrm{a}}$ \\
\hline$\beta$-Carophyllene & $\beta$-car & $0.7 \pm 0.0^{\mathrm{b}}$ & $0.6 \pm 0.6^{\mathrm{ab}}$ & $0.6 \pm 0.0^{\mathrm{b}}$ & $0.3 \pm 0.0^{\mathrm{a}}$ & $1.0 \pm 0.0^{\mathrm{b}}$ & $1.0 \pm 0.0^{\mathrm{b}}$ & $1.4 \pm 1.3^{\mathrm{bc}}$ & $0.8 \pm 0.0^{\mathrm{b}}$ & $1.9 \pm 0.0^{\mathrm{c}}$ & $0.4 \pm 0.0^{\mathrm{a}}$ & $1.0 \pm 0.0^{\mathrm{b}}$ \\
\hline Terpinene-4-ol & ter-4-ol & $\operatorname{tr}^{\mathrm{a}}$ & $\operatorname{tr}^{\mathrm{a}}$ & $\operatorname{tr}^{\mathrm{a}}$ & $0.2 \pm 0.0^{\mathrm{a}}$ & $0.2 \pm 0.0^{\mathrm{a}}$ & $0.1 \pm 0.0^{\mathrm{a}}$ & $0.2 \pm 0.5^{\mathrm{a}}$ & $0.1 \pm 0.0^{\mathrm{a}}$ & $0.3 \pm 0.0^{\mathrm{a}}$ & $0.1 \pm 0.0^{\mathrm{a}}$ & $0.1 \pm 0.0^{\mathrm{a}}$ \\
\hline Aromadendrene & aro & $0.2 \pm 0.0^{\mathrm{a}}$ & $0.2 \pm 0.0^{\mathrm{a}}$ & $0.1 \pm 0.0^{\mathrm{a}}$ & $4.9 \pm 2.1^{\mathrm{e}}$ & $3.8 \pm 0.0^{\mathrm{de}}$ & $3.8 \pm 0.0^{\mathrm{de}}$ & $0.4 \pm 0.5^{\mathrm{a}}$ & $1.8 \pm 0.0^{\mathrm{bc}}$ & $3.2 \pm 0.0^{\mathrm{cd}}$ & $1.2 \pm 0.0^{\mathrm{ab}}$ & $0.5 \pm 0.6^{\mathrm{ab}}$ \\
\hline allo-Aromadendrene & allo-aro & $0.1 \pm 0.0^{\mathrm{a}}$ & $0.2 \pm 0.0^{\mathrm{a}}$ & $0.1 \pm 0.0^{\mathrm{a}}$ & $0.6 \pm 0.0^{\mathrm{c}}$ & $0.6 \pm 0.0^{\mathrm{c}}$ & $0.6 \pm 0.0^{\mathrm{c}}$ & $0.2 \pm 0.0^{\mathrm{a}}$ & $0.5 \pm 0.7^{\mathrm{b}}$ & $0.9 \pm 0.0^{\mathrm{c}}$ & $0.4 \pm 0.0^{\mathrm{a}}$ & $0.2 \pm 0.0^{\mathrm{a}}$ \\
\hline$t r$-Pinocarveol & tr-pin & $0.7 \pm 0.5^{\mathrm{a}}$ & $0.9 \pm 0.0^{\mathrm{a}}$ & $0.9 \pm 1.4^{\mathrm{a}}$ & $10.0 \pm 2.5^{\mathrm{c}}$ & $3.7 \pm 0.0^{\mathrm{b}}$ & $9.3 \pm 1.2^{\mathrm{c}}$ & $1.0 \pm 0.5^{\mathrm{a}}$ & $1.3 \pm 0.0^{\mathrm{a}}$ & $1.8 \pm 0.0^{\mathrm{ab}}$ & $1.5 \pm 0.0^{\mathrm{ab}}$ & $1.1 \pm 0.5^{\mathrm{a}}$ \\
\hline$\alpha$-Terpinyl acetate & $\alpha$-ter acet & $2.6 \pm 0.6^{\mathrm{cd}}$ & $2.1 \pm 0.6^{\mathrm{cd}}$ & $2.0 \pm 2.0^{\mathrm{bc}}$ & $\operatorname{tr}^{\mathrm{a}}$ & $-^{\mathrm{a}}$ & $-{ }^{\mathrm{a}}$ & $2.9 \pm 0.8^{\mathrm{d}}$ & $0.9 \pm 0.0^{\mathrm{b}}$ & $0.7 \pm 0.0^{\mathrm{b}}$ & $0.9 \pm 0.0^{\mathrm{b}}$ & $2.8 \pm 1.0^{\mathrm{d}}$ \\
\hline$\alpha$-Terpineol & $\alpha$-ter & $8.8 \pm 1.1^{\mathrm{e}}$ & $7.5 \pm 0.6^{\mathrm{de}}$ & $6.4 \pm 0.7^{\mathrm{d}}$ & $0.7 \pm 0.0^{\mathrm{ac}}$ & $0.9 \pm 0.0^{\mathrm{a}-\mathrm{c}}$ & $0.5 \pm 0.6^{\mathrm{a}}$ & $2.1 \pm 0.8^{\mathrm{c}}$ & $0.8 \pm 0.7^{\mathrm{ab}}$ & $0.5 \pm 0.0^{\mathrm{a}}$ & $0.2 \pm 0.0^{\mathrm{a}}$ & $1.8 \pm 1.0^{\mathrm{bc}}$ \\
\hline Bicyclogermacene & & $\operatorname{tr}^{\mathrm{a}}$ & - & a & $0.2 \pm 0.2^{\mathrm{a}}$ & $0.5 \pm 0.0^{\mathrm{a}}$ & $0.1 \pm 0.0^{\mathrm{a}}$ & $1.6 \pm 1.7^{\mathrm{a}}$ & $0.6 \pm 0.7^{\mathrm{a}}$ & $3.1 \pm 0.0^{\mathrm{b}}$ & $0.4 \pm 0.0^{\mathrm{a}}$ & $1.1 \pm 1.4^{\mathrm{a}}$ \\
\hline trans-p-Mentha-1(7).8dien-2-ol & tr-p-men & $0.7 \pm 0.5^{\mathrm{b}}$ & $0.8 \pm 0.0^{\mathrm{b}}$ & $0.9 \pm 0.0^{\mathrm{b}}$ & $0.4 \pm 0.0^{\mathrm{a}}$ & $0.2 \pm 0.0^{\mathrm{a}}$ & $0.4 \pm 0.0^{\mathrm{a}}$ & $0.2 \pm 0.0^{\mathrm{a}}$ & $0.8 \pm 0.0^{\mathrm{b}}$ & $0.8 \pm 0.0^{\mathrm{b}}$ & $1.0 \pm 0.0^{\mathrm{b}}$ & $0.4 \pm 0.5^{\mathrm{a}}$ \\
\hline cis-p-Mentha-1(7).8dien-2-ol & cis-p-men & $0.7 \pm 0.5^{\mathrm{b}}$ & $0.9 \pm 0.0^{\mathrm{b}}$ & $0.9 \pm 0.0^{\mathrm{b}}$ & $0.3 \pm 0.0^{\mathrm{a}}$ & $0.2 \pm 0.0^{\mathrm{a}}$ & $0.4 \pm 0.0^{\mathrm{a}}$ & $0.3 \pm 0.0^{\mathrm{a}}$ & $0.8 \pm 0.0^{\mathrm{b}}$ & $0.8 \pm 0.0^{\mathrm{b}}$ & $0.9 \pm 0.0^{\mathrm{b}}$ & $0.4 \pm 0.0^{\mathrm{a}}$ \\
\hline Epiglobulol & epi & $0.1 \pm 0.0^{\mathrm{a}}$ & $0.1 \pm 0.0^{\mathrm{a}}$ & $\operatorname{tr}^{\mathrm{a}}$ & $1.3 \pm 0.6^{\mathrm{c}}$ & $0.9 \pm 0.0^{\mathrm{bc}}$ & $1.1 \pm 0.0^{\mathrm{bc}}$ & $0.1 \pm 0.0^{\mathrm{a}}$ & $0.5 \pm 0.7^{\mathrm{ab}}$ & $0.7 \pm 0.0^{\mathrm{bc}}$ & $0.3 \pm 0.0^{\mathrm{a}}$ & $0.1 \pm 0.0^{\mathrm{a}}$ \\
\hline Globulol & glo & $0.5 \pm 0.0^{\mathrm{ab}}$ & $0.6 \pm 0.0^{\mathrm{ab}}$ & $0.3 \pm 0.0^{\mathrm{a}}$ & $5.7 \pm 1.3^{\mathrm{d}}$ & $4.5 \pm 0.0^{\mathrm{d}}$ & $5.3 \pm 0.6^{\mathrm{d}}$ & $0.9 \pm 0.5^{\mathrm{ab}}$ & $3.0 \pm 0.0^{\mathrm{c}}$ & $4.7 \pm 0.0^{\mathrm{d}}$ & $2.1 \pm 0.0^{\mathrm{bc}}$ & $1.0 \pm 0.8^{\mathrm{ab}}$ \\
\hline Viridiflorol & vir & $0.2 \pm 0.0^{\mathrm{a}}$ & $0.4 \pm 0.0^{\mathrm{a}}$ & $0.2 \pm 0.0^{\mathrm{a}}$ & $0.9 \pm 0 .{ }^{\mathrm{b}} 0$ & $1.0 \pm 0.0^{\mathrm{b}}$ & $0.9 \pm 0.0^{\mathrm{b}}$ & $0.5 \pm 0.5^{\mathrm{a}}$ & $0.7 \pm 0.0^{\mathrm{b}}$ & $1.8 \pm 0.0^{\mathrm{c}}$ & $0.5 \pm 0.0^{\mathrm{b}}$ & $0.5 \pm 0.5^{\mathrm{a}}$ \\
\hline Guaoil & gua & $0.1 \pm 0.0^{\mathrm{a}}$ & $0.1 \pm 0.0^{\mathrm{a}}$ & $0.1 \pm 0.0^{\mathrm{a}}$ & $0.5 \pm 0.6^{\mathrm{b}}$ & $0.5 \pm 0.0^{\mathrm{b}}$ & $0.5 \pm 0.0^{\mathrm{b}}$ & $0.2 \pm 0.0^{\mathrm{a}}$ & $0.3 \pm 0.0^{\mathrm{a}}$ & $0.6 \pm 0.0^{\mathrm{b}}$ & $0.2 \pm 0.0^{\mathrm{a}}$ & $0.2 \pm 0.0^{\mathrm{a}}$ \\
\hline Eremophilene & ere & $0.1 \pm 0.0^{\mathrm{a}}$ & $0.2 \pm 0.0^{\mathrm{a}}$ & $0.1 \pm 0.0^{\mathrm{a}}$ & $0.5 \pm 0.5^{\mathrm{a}}$ & $0.6 \pm 0.0^{\mathrm{b}}$ & $0.4 \pm 0.0^{\mathrm{a}}$ & $0.2 \pm 0.0^{\mathrm{a}}$ & $0.3 \pm 0.0^{\mathrm{a}}$ & $0.7 \pm 0.0^{\mathrm{b}}$ & $0.3 \pm 0.0^{\mathrm{a}}$ & $0.2 \pm 0.0^{\mathrm{a}}$ \\
\hline Spathulenol & spa & $0.2 \pm 0.0^{\mathrm{a}}$ & $0.3 \pm 0.0^{\mathrm{a}}$ & $0.2 \pm 0.0^{\mathrm{a}}$ & $0.5 \pm 0.5^{\mathrm{a}}$ & $1.1 \pm 0.0^{\mathrm{b}}$ & $1.1 \pm 0.0^{\mathrm{b}}$ & $0.5 \pm 0.5$ & $0.8 \pm 0.7^{\mathrm{ab}}$ & $1.3 \pm 0.0^{\mathrm{b}}$ & $0.5 \pm 0.0^{\mathrm{a}}$ & $0.6 \pm 0.6^{\mathrm{ab}}$ \\
\hline iso-Spathulenol & Iso-spa & - & - & - & - & - & - & - & - & $1.1 \pm 0.0^{\mathrm{b}}$ & - & $0.3 \pm 0.5^{\mathrm{a}}$ \\
\hline$\beta$-Eudesmol & $\beta$-eud & $0.1 \pm 0.0^{\mathrm{a}}$ & $0.1 \pm 0.0^{\mathrm{a}}$ & $0.1 \pm 0.0^{\mathrm{a}}$ & $0.1 \pm 0.0^{\mathrm{a}}$ & $0.1 \pm 0.0^{\mathrm{a}}$ & $0.5 \pm 0.0^{\mathrm{a}}$ & $0.3 \pm 0.5^{\mathrm{a}}$ & $0.5 \pm 0.7^{\mathrm{a}}$ & - & $0.4 \pm 0.0^{\mathrm{a}}$ & $0.1 \pm 0.5^{\mathrm{a}}$ \\
\hline
\end{tabular}

Value followed by the different letter in same vertical column is significantly different according to Duncan's multiple range test $(\mathrm{P}<0.05)$ 
3.3 Principal Component Analysis (PCA) and Hierarchical Cluster Analysis (HCA)

To evaluate the effect of the zone of the harvest (arboreta) on the essential oil chemical composition of the studied Eucalyptus species populations, 24 compounds with contents in the essential oils of minimum $0.5 \%$ in at last one species (Table 3), were selected for the PCA and the HCA. The contents of the selected oil components were significantly different between the species $(\mathrm{p}<0.05)$. The PCA horizontal axis explained $43.04 \%$ of the total variance and the vertical axis a further $15.76 \%$ (Figure 1). The HCA (Figure 2) based on the Euclidean distances between groups indicated three groups of species (A, B, and $\mathrm{C}$ ), identified by their essential oil chemotypes with a dissimilarity $>15$ (Figure 2). Group $\mathrm{C}$ was further divided into two Subgroups $(\mathrm{C} 1$ and $\mathrm{C} 2)$ within a dissimilarity $>5$.

Group A reduced to E. sideroxylon from Jbel Abderrahman arboreta, correlated positively with the two axes. It stands out forming a separate group in both HCA and PCA analysis. The essential oil of which was distinguished from the other provennces by the highest mean percentages in bicyclogermacrene $(3.1 \pm 0.0 \%)$, isospathulenol $(1.1 \pm 0.0 \%)$, globulol $(4.7 \pm 0.0 \%)$, viridiflorol $(1.8 \pm 0.0 \%)$, eremophilene $(0.7 \pm 0.0 \%)$, guaoil $(0.6 \pm 0.0 \%$, spathulenol $(1.3 \pm 0.0 \%)$, alloaromadendrene $(0.9 \pm 0.0 \%)$. It was also characterized by the lowest content of $\alpha$-pinene $(2.5 \pm 0.0 \%)$ and by its relatively low percentage in trans-pinocarveol $(1.8 \pm 0.0 \%)$. In comparison with the others provenance, The 1,8-cineole content of this oil was relatively medium $(64.2 \pm 0,0 \%)$. It appears that the type of the soil such us sandy clay for hajeb laayoun arboreta had a significant influence on the biosynthesis of the chemical compound and could favourite ones and inhibits the synthesis of the others.

Group B is represented by oils of E. astringens from Korbous, Sejnane and Mrifek arboreta, correlating positively and negatively with both axes. They are characterized by the highest content in $\alpha$-pinene $(30.0 \pm 0.0,14.0 \pm 1.7,21.8 \pm$ $4.7 \%$ respectively), pinocarvone ( $0.8 \pm 0.0,3.7 \pm 0.6,2.9 \pm$ 0.84 ; respectively) trans- pinocarveol $(3.7 \pm 0.0,9.3 \pm 1.2$, $10.0 \pm 2.5 \%$ respectively), aromadendrene $(3.8 \pm 0.0 \%)$ for those from Korbous and Sejnane arboreta and $4.9 \pm 2.1 \%$ for the provenance Mrifek arboreta, epiglobulol $(0.9 \pm 0.0-1.3 \pm$ $0.6 \%$ ), globulol ( $4.5 \pm 0.0, .5 .3 \pm 0.6,5.7 \pm 1.3 \%$; respectively), viridiflorol ( $0.9-1.0 \%)$ They are also characterized by the lowest mean percentages in 1,8-cineole $(39.1 \pm 0.0,47.6 \pm 1.2,40.1 \pm 5.79 \%$; respectively). The variation between these three sources was mainly due to the variation in the content of these compounds, such as $\alpha$-pinene which distinguish essentially the provenance of Korbous and Sejnane, while the content of tr-pinocarveol, pinocarvone and 1,8- cineole was more abundant in oils obtained from Mrifek and Sejnane sources. Sub-group C1, formed by E. sideroxylon from Korbous and Sejnane, their essential oils are characterized by the highest content in 1,8-cineole ( $79.4 \pm 0.0$,
$71.2 \pm 3.6 \%$; respectively) and by lowest mean percentage in $\alpha$-pinene $(2.5 \pm 0.0,2.9 \pm 1.4 \%$; respectively). However they are also characterized by a relatively high levels of $p$-cymene, tr - $p$ - mentha -1 ( 7 ), 8dien -2 -ol, cis - $p$ - mentha -1 ( 7 ), 8 dien -2 -ol and $\beta$-eudesmol.

Subgroup C2 represented by oils of E. sideroxylon of Souiniat and Mrifek arboreta and by those of E. cinerea from Souiniat, Sejnane and Zerniza arboreta. The essential oils of which were characterized by mean percentages of 1,8-cineole relatively comparable to those of the sub-group C1 ( $66.7 \pm 9.1$ to $72.3 \pm$ $2.7 \%$ ). They were characterized also by the highest content in $\alpha$-pinene $(5.1 \pm 0.8-8.3 \pm 6.4 \%)$, limonene $(3.3 \pm 0.0-4.3 \pm 0.8 \%)$ and $\alpha$-terpinyl acetate $(2.0 \pm 2.0-2.9 \pm 0.8 \%)$, while $E$. cinerea representing the second species of this subgroup was distinguished from the other by its highest mean percentage in $\alpha$-terpineol $(6.4 \pm 0.7$ to $8.8 \pm 1.1 \%)$.

Results of this study are in agreement with the findings of previous researches (Li et al., 1994; Li \& Madden, 1995; Bignell et al., 1997; Della Porta et al., 1999; Tsiri et al., 2003; Marzouki et al., 2009), the chemical composition of the essential oils varied significantly within species and environmental conditions. Zrira et al. (1994) found that the mean percentage of 1,8-cineole of E. sideroxylon essential oil varied from $76.9 \%$ for those from Jbilet region to $80.9 \%$ for those obtained from Tekerkoust area. This variation was also proved within the essential oils of $E$. astringens, mainly in the mean percentages of the 1,8 -cineole $(59.3-61.4 \%)$ and $\alpha$ pinene $(4.9-14.3 \%)$ for the provenances of Jbilet and Tekerkoust; respectively. Grbović et al. (2010) confirmed also this variation within some components of the essential oil of $E$. camaldulensis, such as $p$-cymene $(17.38-28.60 \%)$ for the regions of Herceg Novi and Tivat; respectively, $\beta$-pinene $(0.94,11.48 \%)$ for Tivat and Sutmore; respectively, spathulenol $(7.83,14.15 \%)$ for Tivat and Herceg Novi; respectively and cryptone $(4.97,7.25 \%)$ for Kotor and Tivat regions; respectively.

Dunlop et al. (2003) have also demonstrated this variability within E. Bicostata, cultivated in 12 regions in Australia. They found that 1,8-cineole varied signficantly from $44.0 \%$ for leaves collected from Mount area Bryan (B1) to $71.7 \%$ for those from Wittunga (B8) arboreta. It was the same for $\alpha$ pinene $(1.5-19.6 \%)$ for samples collected from wittunga (B9) and Flinders Island (B3) arboreta, respectively and for aromadendrene (5.4-15.2\%) for those obtained from Wittunga (B8) and Mount Bryan (B1) arboreta respectively.

Conclusions

The essential oils obtained from three Eucalyptus species acclimated in six arboreta were characterized. They varied significantly within species and within their origin of harvest. Four chemotypes were defined, with a great variability between them. E. cinerea grown in Pryor Choucha (Sejnane) arboreta was characterized by the highest mean oil yield, 
whereas E. sideroxylon from Korbous and Sejnane arboreta contained the highest mean percentage in 1,8-cineole.

\section{Conflict of interest}

Authors would hereby like to declare that there is no conflict of interests that could possibly arise.

\section{References}

Arango-Bedoya O, Hurtado-Benavides AM, Toro-Suárez I (2012) Effect of origin, harvesting time and leaf age on the yield and content of thymol in essential oils from Lippia origanoides H.B.K. Acta Agronómica 61: 188-194

Batish DR, Setia N, Singh HP, Kohli RK (2004) Phytotoxicity of lemon-scented eucalypt oil and its potential use as a bioherbicide. Crop Protection 23: 1209 - 1214. doi:10.1016/j.cropro.2004.05.009

Batish DR, Singh HP, Kohli RK, Kaur S (2008) Eucalyptus essential oil as a natural pesticide. Forest Ecology and Management 256: $2166 \quad$ - 2174. doi:10.1016/j.foreco.2008.08.008.

Bhatti HN, Iqbal Z, Chatha SAS, Bukhari IH (2007) Variations in Oil Potential and Chemical Composition of Eucalyptus crebra Among Different Districts of Punjab-Pakistan. International Journal of Agriculture \& Biology 9:136-138.

Bignell CM, Dunlop PJ, Brophy JJ (1997) Volatile leaf oils of some south-western and southern Australian species of the genus Eucalyptus (series I). Part XVI: Subgenus Symphyomyrtus, Section Bisectaria, Series Cneorifoliae, Series Porantherae and Series Falcatae. Flavour and Fragrance Journal 12: 261-267. doi: 10.1002/(SICI)10991026(199707)12:4<261::AID-FFJ647>3.0.CO;2-F.

Boukef MK (1986) Médicine traditionnelle et pharmacopée, les plantes dans la médicine traditionnelle tunisienne. Agence de Coopération Culturelle et Technique. ISBN 92-9028-085-9; 1986.

Bugarin D, Grbović S, Orčič D, Mitić-Ćulafić D, KneževićVukčević J, Mimica-Dukić N (2014) Essential Oil of Eucalyptus Gunnii Hook. As a Novel Source of Antioxidant, Antimutagenic and Antibacterial Agents. Molecules 19: 19007-19020. doi: 10.3390/molecules 191119007.

Cermelli C, Fabio A, Fabio G, Quaglio P (2008) Effect of Eucalyptus essential oil on respiratory bacteria and viruses. Current Microbiology 56:89-92. doi: 10.1007/s00284-0079045-0.

Dunlop PJ, Bignell CM, Hibbert DB, Brooker MIH (2003) Use of gas chromatograms of the essential leaf oils of the genus Eucalyptus for taxonomic purposes: $E$. subser. Euglobulares (Blakely). Flavour and Fragrance Journal 18: 162-169. DOI: 10.1002/ffj.1190.

Dunlop PJ, Bignell CM, Brynn Hibbert D (2000) Use of gas chromatograms of essential leaf oils to compare clones of Eucalyptus camaldulensis. Biochemical Systematics and Ecology 28:383-391. doi:10.1016/S0305-1978(99)00067-8.

Elaissi A, Marzoukia H, Medini H, Khouja ML, Farhat F, Lynene F, Harzallah-Skhir F, Chemli R (2010c) Variation in Volatile Leaf Oils of 13 Eucalyptus Species Harvested from Souinet Arboreta (Tunisia). Chemistry \& Biodiversity 7: 909921. doi: $10.1002 / \mathrm{cbdv} .200900229$.

Elaissi A, Medini H, Larbi Khouja M, Simmonds M, Lynene F, Farhat F, Chemli R, Harzallah-Skhiri F (2010) Variation in Volatile Leaf Oils of Eleven Eucalyptus Species Harvested from Korbous Arboreta (Tunisia). Chemistry \& Biodiversity 7: 1841-1854. doi: 10.1002/cbdv.200900381.

Elaissi A, Medini H, Marzouki H, Khouja ML, Lyenne F, Chemli R, Harzallah-Skhiri F (2010b) Variation in Volatile Leaf Oils of Twelve Eucalyptus Species Harvested from Hajeb Layoun Arboreta (Tunisia). Chemistry \& Biodiversity 7: 705-716. doi: 10.1002/cbdv.200900169.

Elaissi A, Rouis Z, Abid Ben Salem N, Mabrouk S, Ben Salem Y, Bel Haj Salah K, Aouni M, Farhat F, Chemli R, HarzallahSkhiri F, Khouja ML (2012) Chemical composition of 8 Eucalyptus species' essential oils and the evaluation of their antibacterial, antifungal and antiviral activities. BMC Complementary and Alternative Medicine 12:81. doi:10.1186/1472-6882-12-81.

Gilles M, Zhao J, An M, Agboola S (2010) Chemical composition and antimicrobial properties of essential oils of three Australian Eucalyptus species. Food Chemistry 119: 731 -737. doi:10.1016/j.foodchem.2009.07.021.

Grbović S, Orčić D, Couladis M, Jovin E, Bugarin D, Balog K, Mimica-Dukić N (2010) Variation Of Essential Oil Composition Of Eucalyptus camaldulensis (Myrtaceae) from the Montengero Coastline. Acta Periodica Technologica 41:151-158. DOI: 10.2298/APT1041151G.

Hassiotis CN, Ntana F, Lazari DM, Poulios S, Vlachonasios KE (2014) Environmental and developmental factors affect essential oil production and quality of Lavandula angustifolia during flowering period. Industrial Crops and Products 62: 359-366. doi:10.1016/j.indcrop.2014.08.048.

Jha R, Regmi R, Simon LS, Lal AA (2014) Efficacy of Eucalyptus essential oil against leaf spot (Alternaria solani) of Solanum melongena L. ARPN Journal of Agricultural and Biological Science 9: 320-322.

Khouja ML, Khaldi A, Rejeb MN (2001) Results of the Eucalyptus introduction trials in Tunisia_,Proceedings of the 
International Conference Eucalyptus in the Mediterranean basin: perspectives and new utilization, October 15-19, 2000, Centro Promozione Pubblicita', Florence, 2001, p. 163.

Kiran Babu GD, Singh B (2009) Simulation of Eucalyptus cinerea oil distillation: A study on optimization of 1,8-cineole production. Biochemical Engineering Journal 44:226-231. doi:10.1016/j.bej.2008.12.012.

Li H, Madden JL (1995) Analysis of leaf oils from a Eucalyptus species trial. Biochemical Systematics and Ecology 23: 167-177. doi:10.1016/0305-1978(94)00087-W.

LI H, Madden JL, Davies NW (1994) Variation in leaf oil of Eucalyptus nitens and E. denticulata. Biochemical Systematics and Ecology 22: 631-640. doi:10.1016/0305-1978(94)90076-0.

Marzouki H, Elaissi A, Khaldi A, Bouzid S, Falconieri D, Marongiu B, Piaras A, Porcedda S (2009) Seasonal and Geographical Variation of Laurus nobilis L. Essential Oil from Tunisia. Open Natural Product Journal 2: 86-91.

Pereira V, Dias C, Vasconcelos MC, Rosa E, Saavedra MJ (2014) Antibacterial activity and synergistic effects between Eucalyptus globulus leaf residues (essential oils and extracts) and antibiotics against several isolates of respiratory tract infections (Pseudomonas aeruginosa). Industrial Crops and Products 52:1-7. doi:10.1016/j.indcrop.2013.09.032.

Pino JA, Marbot R, Quert R, García H (2002) Study of essential oils of Eucalyptus resinifera Smith, E. tereticornis Smith and Corymbia maculata (Hook.) K. D. Hill \& L. A. S. Johnson, grown in Cuba. Flavour and Fragrance Journal 17:114. doi: $10.1002 / f f j .1026$.

Della Porta, G., Porcedda, S., Marongiu, B. and Reverchon, E. (1999), Isolation of eucalyptus oil by supercritical fluid extraction. Flavour and Fragrance Journal 14: 214-218. doi: 10.1002/(SICI)1099-1026(199907/08)14:4<214::AID-

FFJ814>3.0.CO;2-H.
Rassaeifar M, Hosseini N, ANH Hasani, Zandi P, Aghdam AM (2013) Allelopathic effect of Eucalyptus globulus' essential oil on seed germination and seedling establishment of Aamaranthus blitoides and Cyndon dactylon. Trakia Journal of Sciences 11:73-81.

Rossi YE, Palacios SM (2015) Insecticidal toxicity of Eucalyptus cinerea essential oil and 1,8-cineole against Musca domestica and possible uses according to the metabolic response of flies. Industrial Crops and Products 63:133-137. doi:10.1016/j.indcrop.2014.10.019.

Su YC, Ho CL, Wang CL, Wang EIC, Chang ST (2006) Antifungal activities and chemical compositions of essential oils from leaves of four Eucalypts. Taiwan Journal of Forest Science 21:49-61.

Tsiri, D., Kretsi, O., Chinou, I. B. and Spyropoulos, C. G. (2003), Composition of fruit volatiles and annual changes in the volatiles of leaves of Eucalyptus camaldulensis Dehn. growing in Greece. Flavour and Fragrance Journal 18: 244247. doi: $10.1002 /$ ffj. 1220 .

Verdeguer M, Blázquez MA , Boira H (2009) Phytotoxic effects of Lantana camara, Eucalyptus camaldulensis and Eriocephalus africanus essential oils in weeds of Mediterranean summer crops. Biochemical Systematics and Ecology 37:362-369. doi:10.1016/j.bse.2009.06.003.

Wiley Registry of Mass Spectral Data/NIST Spectral Data/CD Rom, 7th edn., John Wiley \& Sons, New York, 1998.

Zrira S, Bessiere JM, Menut C, Elamrani A, Benjilali B (2004), Chemical composition of the essential oil of nine Eucalyptus species growing in Morocco. Flavour and Fragrance Journal 19: 172-175. doi: 10.1002/ffj.1289.

Zrira S, El Khiranti F, Benjilali B (1994) Huiles essentielles de six espèces xérophyles d'Eucalyptus:effet du milieu sur les rendements et la composition chimique. Actes de l'Institut agronomique et vétérinaire (Maroc) 14: 5-9. 